Supporting Information

\title{
High-throughput synthesis, analysis, and optimization of injectable hydrogels for protein delivery
}

Fei Xu, Brandon Corbett, Sydney Bell, Chiyan Zhang, Monika Budi Hartono, Zohreh Jomeh Farsangi, John MacGregor, and Todd Hoare*

Department of Chemical Engineering, McMaster University, 1280 Main Street West, Hamilton, ON L8S 4L8, Canada

* To whom correspondence should be addressed: hoaretr@mcmaster.ca 
Table S1. Compositions of thermoresponsive vs. non-thermoresponsive combinatorial hydrogels

\begin{tabular}{|c|c|c|c|c|}
\hline Number & Group & Label & Components of hydrazide polymer precursors & $\begin{array}{c}\text { Volume of } \\
\text { each } \\
\text { component }\end{array}$ \\
\hline 1 & \multirow{6}{*}{$C_{6}^{1}$} & T1-1 & $\mathrm{PO}_{0} \mathrm{H}_{30}$ & \multirow{6}{*}{$60 \mu \mathrm{L}$} \\
\hline 2 & & T1-2 & $\mathrm{PO}_{10} \mathrm{H}_{30}$ & \\
\hline 3 & & T1-3 & $\mathrm{PO}_{50} \mathrm{H}_{30}$ & \\
\hline 4 & & T1-4 & $\mathrm{PO}_{100} \mathrm{H}_{30}$ & \\
\hline 5 & & $\mathrm{~T} 1-5$ & PNIPAM-Hzd & \\
\hline 6 & & T1-6 & $\mathrm{CMC}_{40}-\mathrm{Hzd}$ & \\
\hline 7 & \multirow{15}{*}{$C_{6}^{2}$} & T2-1 & $\mathrm{PO}_{10} \mathrm{H}_{30}+\mathrm{PO}_{100} \mathrm{H}_{30}$ & \multirow{15}{*}{$30 \mu \mathrm{L}$} \\
\hline 8 & & $\mathrm{~T} 2-2$ & $\mathrm{PO}_{10} \mathrm{H}_{30}+\mathrm{PO}_{0} \mathrm{H}_{30}$ & \\
\hline 9 & & $\mathrm{~T} 2-3$ & $\mathrm{PO}_{10} \mathrm{H}_{30}+\mathrm{PO}_{50} \mathrm{H}_{30}$ & \\
\hline 10 & & T2-4 & $\mathrm{PO}_{10} \mathrm{H}_{30}+$ PNIPAM-Hzd & \\
\hline 11 & & $\mathrm{~T} 2-5$ & $\mathrm{PO}_{10} \mathrm{H}_{30}+\mathrm{CMC}_{40}-\mathrm{Hzd}$ & \\
\hline 12 & & T2-6 & $\mathrm{PO}_{100} \mathrm{H}_{30}+\mathrm{PO}_{0} \mathrm{H}_{30}$ & \\
\hline 13 & & T2-7 & $\mathrm{PO}_{100} \mathrm{H}_{30}+\mathrm{PO}_{50} \mathrm{H}_{30}$ & \\
\hline 14 & & $\mathrm{~T} 2-8$ & $\mathrm{PO}_{100} \mathrm{H}_{30}+$ PNIPAM-Hzd & \\
\hline 15 & & T2-9 & $\mathrm{PO}_{100} \mathrm{H}_{30}+\mathrm{CMC}_{40}-\mathrm{Hzd}$ & \\
\hline 16 & & $\mathrm{~T} 2-10$ & $\mathrm{PO}_{0} \mathrm{H}_{30}+\mathrm{PO}_{50} \mathrm{H}_{30}$ & \\
\hline 17 & & $\mathrm{~T} 2-11$ & $\mathrm{PO}_{0} \mathrm{H}_{30}+$ PNIPAM-Hzd & \\
\hline 18 & & $\mathrm{~T} 2-12$ & $\mathrm{PO}_{0} \mathrm{H}_{30}+\mathrm{CMC}_{40}-\mathrm{Hzd}$ & \\
\hline 19 & & $\mathrm{~T} 2-13$ & $\mathrm{PO}_{50} \mathrm{H}_{30}+$ PNIPAM-Hzd & \\
\hline 20 & & $\mathrm{~T} 2-14$ & $\mathrm{PO}_{50} \mathrm{H}_{30}+\mathrm{CMC}_{40}-\mathrm{Hzd}$ & \\
\hline 21 & & $\mathrm{~T} 2-15$ & PNIPAM-Hzd $+\mathrm{CMC}_{40}-\mathrm{Hzd}$ & \\
\hline 22 & \multirow{20}{*}{$C_{6}^{3}$} & T3-1 & $\mathrm{PO}_{10} \mathrm{H}_{30}+\mathrm{PO}_{100} \mathrm{H}_{30}+\mathrm{PO}_{0} \mathrm{H}_{30}$ & \multirow{20}{*}{$20 \mu \mathrm{L}$} \\
\hline 23 & & T3-2 & $\mathrm{PO}_{10} \mathrm{H}_{30}+\mathrm{PO}_{100} \mathrm{H}_{30}+\mathrm{PO}_{50} \mathrm{H}_{30}$ & \\
\hline 24 & & T3-3 & $\mathrm{PO}_{10} \mathrm{H}_{30}+\mathrm{PO}_{100} \mathrm{H}_{30}+$ PNIPAM-Hzd & \\
\hline 25 & & T3-4 & $\mathrm{PO}_{10} \mathrm{H}_{30}+\mathrm{PO}_{100} \mathrm{H}_{30}+\mathrm{CMC}_{40}-\mathrm{Hzd}$ & \\
\hline 26 & & T3-5 & $\mathrm{PO}_{10} \mathrm{H}_{30}+\mathrm{PO}_{0} \mathrm{H}_{30}+\mathrm{PO}_{50} \mathrm{H}_{30}$ & \\
\hline 27 & & T3-6 & $\mathrm{PO}_{10} \mathrm{H}_{30}+\mathrm{PO}_{0} \mathrm{H}_{30}+$ PNIPAM-Hzd & \\
\hline 28 & & T3-7 & $\mathrm{PO}_{10} \mathrm{H}_{30}+\mathrm{PO}_{0} \mathrm{H}_{30}+\mathrm{CMC}_{40}-\mathrm{Hzd}$ & \\
\hline 29 & & T3-8 & $\mathrm{PO}_{10} \mathrm{H}_{30}+\mathrm{PO}_{50} \mathrm{H}_{30}+$ PNIPAM-Hzd & \\
\hline 30 & & T3-9 & $\mathrm{PO}_{10} \mathrm{H}_{30}+\mathrm{PO}_{50} \mathrm{H}_{30}+\mathrm{CMC}_{40}-\mathrm{Hzd}$ & \\
\hline 31 & & $\mathrm{~T} 3-10$ & $\mathrm{PO}_{10} \mathrm{H}_{30}+$ PNIPAM-Hzd $+\mathrm{CMC}_{40}-\mathrm{Hzd}$ & \\
\hline 32 & & T3-11 & $\mathrm{PO}_{100} \mathrm{H}_{30}+\mathrm{PO}_{0} \mathrm{H}_{30}+\mathrm{PO}_{50} \mathrm{H}_{30}$ & \\
\hline 33 & & T3-12 & $\mathrm{PO}_{100} \mathrm{H}_{30}+\mathrm{PO}_{0} \mathrm{H}_{30}+$ PNIPAM-Hzd & \\
\hline 34 & & $\mathrm{~T} 3-13$ & $\mathrm{PO}_{100} \mathrm{H}_{30}+\mathrm{PO}_{0} \mathrm{H}_{30}+\mathrm{CMC}_{40}-\mathrm{Hzd}$ & \\
\hline 35 & & $\mathrm{~T} 3-14$ & $\mathrm{PO}_{100} \mathrm{H}_{30}+\mathrm{PO}_{50} \mathrm{H}_{30}+$ PNIPAM-Hzd & \\
\hline 36 & & $\mathrm{~T} 3-15$ & $\mathrm{PO}_{100} \mathrm{H}_{30}+\mathrm{PO}_{50} \mathrm{H}_{30}+\mathrm{CMC}_{40}-\mathrm{Hzd}$ & \\
\hline 37 & & T3-16 & $\mathrm{PO}_{100} \mathrm{H}_{30}+\mathrm{PNIPAM}-\mathrm{Hzd}+\mathrm{CMC}_{40}-\mathrm{Hzd}$ & \\
\hline 38 & & T3-17 & $\mathrm{PO}_{0} \mathrm{H}_{30}+\mathrm{PO}_{50} \mathrm{H}_{30}+$ PNIPAM-Hzd & \\
\hline 39 & & T3-18 & $\mathrm{PO}_{0} \mathrm{H}_{30}+\mathrm{PO}_{50} \mathrm{H}_{30}+\mathrm{CMC}_{40}-\mathrm{Hzd}$ & \\
\hline 40 & & T3-19 & $\mathrm{PO}_{0} \mathrm{H}_{30}+\mathrm{PNIPAM}-\mathrm{Hzd}+\mathrm{CMC}_{40}-\mathrm{Hzd}$ & \\
\hline 41 & & $\mathrm{~T} 3-20$ & $\mathrm{PO}_{50} \mathrm{H}_{30}+$ PNIPAM-Hzd $+\mathrm{CMC}_{40}-\mathrm{Hzd}$ & \\
\hline
\end{tabular}




\begin{tabular}{|c|c|c|c|c|}
\hline 42 & \multirow{15}{*}{$C_{6}^{4}$} & T4-1 & $\mathrm{PO}_{10} \mathrm{H}_{30}+\mathrm{PO}_{100} \mathrm{H}_{30}+\mathrm{PO}_{0} \mathrm{H}_{30}+\mathrm{PO}_{50} \mathrm{H}_{30}$ & \multirow{15}{*}{$15 \mu \mathrm{L}$} \\
\hline 43 & & $\mathrm{~T} 4-2$ & $\mathrm{PO}_{10} \mathrm{H}_{30}+\mathrm{PO}_{100} \mathrm{H}_{30}+\mathrm{PO}_{0} \mathrm{H}_{30}+$ PNIPAM-Hzd & \\
\hline 44 & & $\mathrm{~T} 4-3$ & $\mathrm{PO}_{10} \mathrm{H}_{30}+\mathrm{PO}_{100} \mathrm{H}_{30}+\mathrm{PO}_{0} \mathrm{H}_{30}+\mathrm{CMC}_{40}-\mathrm{Hzd}$ & \\
\hline 45 & & T4-4 & $\mathrm{PO}_{10} \mathrm{H}_{30}+\mathrm{PO}_{100} \mathrm{H}_{30}+\mathrm{PO}_{50} \mathrm{H}_{30}+$ PNIPAM-Hzd & \\
\hline 46 & & T4-5 & $\mathrm{PO}_{10} \mathrm{H}_{30}+\mathrm{PO}_{100} \mathrm{H}_{30}+\mathrm{PO}_{50} \mathrm{H}_{30}+\mathrm{CMC}_{40}-\mathrm{Hzd}$ & \\
\hline 47 & & T4-6 & $\mathrm{PO}_{10} \mathrm{H}_{30}+\mathrm{PO}_{100} \mathrm{H}_{30}+$ PNIPAM-Hzd $+\mathrm{CMC}_{40}-\mathrm{Hzd}$ & \\
\hline 48 & & T4-7 & $\mathrm{PO}_{10} \mathrm{H}_{30}+\mathrm{PO}_{0} \mathrm{H}_{30}+\mathrm{PO}_{50} \mathrm{H}_{30}+$ PNIPAM-Hzd & \\
\hline 49 & & T4-8 & $\mathrm{PO}_{10} \mathrm{H}_{30}+\mathrm{PO}_{0} \mathrm{H}_{30}+\mathrm{PO}_{50} \mathrm{H}_{30}+\mathrm{CMC}_{40}-\mathrm{Hzd}$ & \\
\hline 50 & & T4-9 & $\mathrm{PO}_{10} \mathrm{H}_{30}+\mathrm{PO}_{0} \mathrm{H}_{30}+$ PNIPAM-Hzd $+\mathrm{CMC}_{40}-\mathrm{Hzd}$ & \\
\hline 51 & & $\mathrm{~T} 4-10$ & $\mathrm{PO}_{10} \mathrm{H}_{30}+\mathrm{PO}_{50} \mathrm{H}_{30}+$ PNIPAM-Hzd $+\mathrm{CMC}_{40}-\mathrm{Hzd}$ & \\
\hline 52 & & T4-11 & $\mathrm{PO}_{100} \mathrm{H}_{30}+\mathrm{PO}_{0} \mathrm{H}_{30}+\mathrm{PO}_{50} \mathrm{H}_{30}+$ PNIPAM-Hzd & \\
\hline 53 & & $\mathrm{~T} 4-12$ & $\mathrm{PO}_{100} \mathrm{H}_{30}+\mathrm{PO}_{0} \mathrm{H}_{30}+\mathrm{PO}_{50} \mathrm{H}_{30}+\mathrm{CMC}_{40}-\mathrm{Hzd}$ & \\
\hline 54 & & T4-13 & $\mathrm{PO}_{100} \mathrm{H}_{30}+\mathrm{PO}_{0} \mathrm{H}_{30}+$ PNIPAM-Hzd $+\mathrm{CMC}_{40}-\mathrm{Hzd}$ & \\
\hline 55 & & T4-14 & $\mathrm{PO}_{100} \mathrm{H}_{30}+\mathrm{PO}_{50} \mathrm{H}_{30}+$ PNIPAM-Hzd $+\mathrm{CMC}_{40}-\mathrm{Hzd}$ & \\
\hline 56 & & T4-15 & $\mathrm{PO}_{0} \mathrm{H}_{30}+\mathrm{PO}_{50} \mathrm{H}_{30}+$ PNIPAM-Hzd $+\mathrm{CMC}_{40}-\mathrm{Hzd}$ & \\
\hline 57 & \multirow{6}{*}{$C_{6}^{5}$} & T5-1 & $\mathrm{PO}_{10} \mathrm{H}_{30}+\mathrm{PO}_{100} \mathrm{H}_{30}+\mathrm{PO}_{0} \mathrm{H}_{30}+\mathrm{PO}_{50} \mathrm{H}_{30}+$ PNIPAM-Hzd & \multirow{6}{*}{$12 \mu \mathrm{L}$} \\
\hline 58 & & T5-2 & $\mathrm{PO}_{10} \mathrm{H}_{30}+\mathrm{PO}_{100} \mathrm{H}_{30}+\mathrm{PO}_{0} \mathrm{H}_{30}+\mathrm{PO}_{50} \mathrm{H}_{30}+\mathrm{CMC}_{40}-\mathrm{Hzd}$ & \\
\hline 59 & & $\mathrm{~T} 5-3$ & $\mathrm{PO}_{10} \mathrm{H}_{30}+\mathrm{PO}_{100} \mathrm{H}_{30}+\mathrm{PO}_{0} \mathrm{H}_{30}+$ PNIPAM-Hzd $+\mathrm{CMC}_{40}-\mathrm{Hzd}$ & \\
\hline 60 & & T5-4 & $\mathrm{PO}_{10} \mathrm{H}_{30}+\mathrm{PO}_{100} \mathrm{H}_{30}+\mathrm{PO}_{50} \mathrm{H}_{30}+$ PNIPAM-Hzd $+\mathrm{CMC}_{40}-\mathrm{Hzd}$ & \\
\hline 61 & & $\mathrm{~T} 5-5$ & $\mathrm{PO}_{10} \mathrm{H}_{30}+\mathrm{PO}_{0} \mathrm{H}_{30}+\mathrm{PO}_{50} \mathrm{H}_{30}+$ PNIPAM-Hzd $+\mathrm{CMC}_{40}-\mathrm{Hzd}$ & \\
\hline 62 & & T5-6 & $\mathrm{PO}_{100} \mathrm{H}_{30}+\mathrm{PO}_{0} \mathrm{H}_{30}+\mathrm{PO}_{50} \mathrm{H}_{30}+$ PNIPAM-Hzd $+\mathrm{CMC}_{40}-\mathrm{Hzd}$ & \\
\hline 63 & $C_{6}^{6}$ & T6-1 & $\begin{array}{l}\mathrm{PO}_{10} \mathrm{H}_{30}+\mathrm{PO}_{100} \mathrm{H}_{30}+\mathrm{PO}_{0} \mathrm{H}_{30}+\mathrm{PO}_{50} \mathrm{H}_{30}+\text { PNIPAM-Hzd }+ \\
\mathrm{CMC}_{40} \text {-Hzd }\end{array}$ & $10 \mu \mathrm{L}$ \\
\hline
\end{tabular}

* The combinations of hydrazide polymers listed above were each mixed with a single aldehyde precursor polymer $\left(\mathrm{PO}_{10} \mathrm{~A}_{30}, 60 \mu \mathrm{L}\right)$ to facilitate gelation. The total volume of each gel was 120 $\mu \mathrm{L}$. CMC-Hzd was added at a concentration of $40 \mathrm{mg} / \mathrm{mL}$ 
Table S2. Compositions of charged vs. neutral combinatorial hydrogels

\begin{tabular}{|c|c|c|c|c|}
\hline Number & Group & Label & Components of hydrazide polymer precursors & $\begin{array}{c}\text { Volume of } \\
\text { each } \\
\text { component }\end{array}$ \\
\hline 1 & \multirow{6}{*}{$C_{6}^{1}$} & C1-1 & $\mathrm{PO}_{100} \mathrm{H}_{30}$ & \multirow{6}{*}{$60 \mu \mathrm{L}$} \\
\hline 2 & & $\mathrm{C} 1-2$ & $\mathrm{PO}_{100} \mathrm{H}_{30} \mathrm{C}_{20}$ & \\
\hline 3 & & $\mathrm{C} 1-3$ & $\mathrm{PO}_{100} \mathrm{H}_{30} \mathrm{~A}_{20}$ & \\
\hline 4 & & $\mathrm{C} 1-4$ & $\mathrm{CMC}_{20}-\mathrm{Hzd}$ & \\
\hline 5 & & $\mathrm{C} 1-5$ & Dextran-Hzd & \\
\hline 6 & & $\mathrm{C} 1-6$ & Chitosan-Hzd & \\
\hline 7 & \multirow{15}{*}{$C_{6}^{2}$} & $\mathrm{C} 2-1$ & $\mathrm{PO}_{100} \mathrm{H}_{30}+\mathrm{PO}_{100} \mathrm{H}_{30} \mathrm{C}_{20}$ & \multirow{15}{*}{$30 \mu \mathrm{L}$} \\
\hline 8 & & $\mathrm{C} 2-2$ & $\mathrm{PO}_{100} \mathrm{H}_{30}+\mathrm{PO}_{100} \mathrm{H}_{30} \mathrm{~A}_{20}$ & \\
\hline 9 & & $\mathrm{C} 2-3$ & $\mathrm{PO}_{100} \mathrm{H}_{30}+\mathrm{CMC}_{20}-\mathrm{Hzd}$ & \\
\hline 10 & & $\mathrm{C} 2-4$ & $\mathrm{PO}_{100} \mathrm{H}_{30}+$ Dextran-Hzd & \\
\hline 11 & & $\mathrm{C} 2-5$ & $\mathrm{PO}_{100} \mathrm{H}_{30}+$ Chitosan-Hzd & \\
\hline 12 & & $\mathrm{C} 2-6$ & $\mathrm{PO}_{100} \mathrm{H}_{30} \mathrm{C}_{20}+\mathrm{PO}_{100} \mathrm{H}_{30} \mathrm{~A}_{20}$ & \\
\hline 13 & & $\mathrm{C} 2-7$ & $\mathrm{PO}_{100} \mathrm{H}_{30} \mathrm{C}_{20}+\mathrm{CMC}_{20}-\mathrm{Hzd}$ & \\
\hline 14 & & $\mathrm{C} 2-8$ & $\mathrm{PO}_{100} \mathrm{H}_{30} \mathrm{C}_{20}+$ Dextran-Hzd & \\
\hline 15 & & $\mathrm{C} 2-9$ & $\mathrm{PO}_{100} \mathrm{H}_{30} \mathrm{C}_{20}+$ Chitosan-Hzd & \\
\hline 16 & & $\mathrm{C} 2-10$ & $\mathrm{PO}_{100} \mathrm{H}_{30} \mathrm{~A}_{20}+\mathrm{CMC}_{20}-\mathrm{Hzd}$ & \\
\hline 17 & & $\mathrm{C} 2-11$ & $\mathrm{PO}_{100} \mathrm{H}_{30} \mathrm{~A}_{20}+$ Dextran-Hzd & \\
\hline 18 & & $\mathrm{C} 2-12$ & $\mathrm{PO}_{100} \mathrm{H}_{30} \mathrm{~A}_{20}+$ Chitosan-Hzd & \\
\hline 19 & & $\mathrm{C} 2-13$ & $\mathrm{CMC}_{20}-\mathrm{Hzd}+$ Dextran-Hzd & \\
\hline 20 & & $\mathrm{C} 2-14$ & $\mathrm{CMC}_{20}-\mathrm{Hzd}+$ Chitosan-Hzd & \\
\hline 21 & & $\mathrm{C} 2-15$ & Dextran-Hzd + Chitosan-Hzd & \\
\hline 22 & \multirow{20}{*}{$C_{6}^{3}$} & C3-1 & $\mathrm{PO}_{100} \mathrm{H}_{30}+\mathrm{PO}_{100} \mathrm{H}_{30} \mathrm{C}_{20}+\mathrm{PO}_{100} \mathrm{H}_{30} \mathrm{~A}_{20}$ & \multirow{20}{*}{$20 \mu \mathrm{L}$} \\
\hline 23 & & $\mathrm{C} 3-2$ & $\mathrm{PO}_{100} \mathrm{H}_{30}+\mathrm{PO}_{100} \mathrm{H}_{30} \mathrm{C}_{20}+\mathrm{CMC}_{20}-\mathrm{Hzd}$ & \\
\hline 24 & & $\mathrm{C} 3-3$ & $\mathrm{PO}_{100} \mathrm{H}_{30}+\mathrm{PO}_{100} \mathrm{H}_{30} \mathrm{C}_{20}+$ Dextran-Hzd & \\
\hline 25 & & $\mathrm{C} 3-4$ & $\mathrm{PO}_{100} \mathrm{H}_{30}+\mathrm{PO}_{100} \mathrm{H}_{30} \mathrm{C}_{20}+$ Chitosan-Hzd & \\
\hline 26 & & $\mathrm{C} 3-5$ & $\mathrm{PO}_{100} \mathrm{H}_{30}+\mathrm{PO}_{100} \mathrm{H}_{30} \mathrm{~A}_{20}+\mathrm{CMC}_{20}-\mathrm{Hzd}$ & \\
\hline 27 & & $\mathrm{C} 3-6$ & $\mathrm{PO}_{100} \mathrm{H}_{30}+\mathrm{PO}_{100} \mathrm{H}_{30} \mathrm{~A}_{20}+$ Dextran-Hzd & \\
\hline 28 & & $\mathrm{C} 3-7$ & $\mathrm{PO}_{100} \mathrm{H}_{30}+\mathrm{PO}_{100} \mathrm{H}_{30} \mathrm{~A}_{20}+$ Chitosan-Hzd & \\
\hline 29 & & $\mathrm{C} 3-8$ & $\mathrm{PO}_{100} \mathrm{H}_{30}+\mathrm{CMC}_{20}-\mathrm{Hzd}+$ Dextran-Hzd & \\
\hline 30 & & C3-9 & $\mathrm{PO}_{100} \mathrm{H}_{30}+\mathrm{CMC}_{20}-\mathrm{Hzd}+$ Chitosan-Hzd & \\
\hline 31 & & $\mathrm{C} 3-10$ & $\mathrm{PO}_{100} \mathrm{H}_{30}+$ Dextran-Hzd + Chitosan-Hzd & \\
\hline 32 & & $\mathrm{C} 3-11$ & $\mathrm{PO}_{100} \mathrm{H}_{30} \mathrm{C}_{20}+\mathrm{PO}_{100} \mathrm{H}_{30} \mathrm{~A}_{20}+\mathrm{CMC}_{20}-\mathrm{Hzd}$ & \\
\hline 33 & & $\mathrm{C} 3-12$ & $\mathrm{PO}_{100} \mathrm{H}_{30} \mathrm{C}_{20}+\mathrm{PO}_{100} \mathrm{H}_{30} \mathrm{~A}_{20}+$ Dextran-Hzd & \\
\hline 34 & & $\mathrm{C} 3-13$ & $\mathrm{PO}_{100} \mathrm{H}_{30} \mathrm{C}_{20}+\mathrm{PO}_{100} \mathrm{H}_{30} \mathrm{~A}_{20}+$ Chitosan-Hzd & \\
\hline 35 & & C3-14 & $\mathrm{PO}_{100} \mathrm{H}_{30} \mathrm{C}_{20}+\mathrm{CMC}_{20}-\mathrm{Hzd}+$ Dextran-Hzd & \\
\hline 36 & & $\mathrm{C} 3-15$ & $\mathrm{PO}_{100} \mathrm{H}_{30} \mathrm{C}_{20}+\mathrm{CMC}_{20}-\mathrm{Hzd}+$ Chitosan-Hzd & \\
\hline 37 & & $\mathrm{C} 3-16$ & $\mathrm{PO}_{100} \mathrm{H}_{30} \mathrm{C}_{20}+$ Dextran-Hzd + Chitosan-Hzd & \\
\hline 38 & & $\mathrm{C} 3-17$ & $\mathrm{PO}_{100} \mathrm{H}_{30} \mathrm{~A}_{20}+\mathrm{CMC}_{20}-\mathrm{Hzd}+$ Dextran-Hzd & \\
\hline 39 & & C3-18 & $\mathrm{PO}_{100} \mathrm{H}_{30} \mathrm{~A}_{20}+\mathrm{CMC}_{20}-\mathrm{Hzd}+$ Chitosan-Hzd & \\
\hline 40 & & $\mathrm{C} 3-19$ & $\mathrm{PO}_{100} \mathrm{H}_{30} \mathrm{~A}_{20}+$ Dextran-Hzd + Chitosan-Hzd & \\
\hline 41 & & $\mathrm{C} 3-20$ & $\mathrm{CMC}_{20}-\mathrm{Hzd}+$ Dextran-Hzd + Chitosan-Hzd & \\
\hline
\end{tabular}




\begin{tabular}{|c|c|c|c|c|}
\hline 42 & \multirow{15}{*}{$C_{6}^{4}$} & C4-1 & $\mathrm{PO}_{100} \mathrm{H}_{30}+\mathrm{PO}_{100} \mathrm{H}_{30} \mathrm{C}_{20}+\mathrm{PO}_{100} \mathrm{H}_{30} \mathrm{~A}_{20}+\mathrm{CMC}_{20}-\mathrm{Hzd}$ & \multirow{15}{*}{$15 \mu \mathrm{L}$} \\
\hline 43 & & $\mathrm{C} 4-2$ & $\mathrm{PO}_{100} \mathrm{H}_{30}+\mathrm{PO}_{100} \mathrm{H}_{30} \mathrm{C}_{20}+\mathrm{PO}_{100} \mathrm{H}_{30} \mathrm{~A}_{20}+$ Dextran-Hzd & \\
\hline 44 & & $\mathrm{C} 4-3$ & $\mathrm{PO}_{100} \mathrm{H}_{30}+\mathrm{PO}_{100} \mathrm{H}_{30} \mathrm{C}_{20}+\mathrm{PO}_{100} \mathrm{H}_{30} \mathrm{~A}_{20}+$ Chitosan-Hzd & \\
\hline 45 & & $\mathrm{C} 4-4$ & $\mathrm{PO}_{100} \mathrm{H}_{30}+\mathrm{PO}_{100} \mathrm{H}_{30} \mathrm{C}_{20}+\mathrm{CMC}_{20}-\mathrm{Hzd}+$ Dextran-Hzd & \\
\hline 46 & & $\mathrm{C} 4-5$ & $\mathrm{PO}_{100} \mathrm{H}_{30}+\mathrm{PO}_{100} \mathrm{H}_{30} \mathrm{C}_{20}+\mathrm{CMC}_{20}-\mathrm{Hzd}+$ Chitosan-Hzd & \\
\hline 47 & & $\mathrm{C} 4-6$ & $\mathrm{PO}_{100} \mathrm{H}_{30}+\mathrm{PO}_{100} \mathrm{H}_{30} \mathrm{C}_{20}+$ Dextran-Hzd + Chitosan-Hzd & \\
\hline 48 & & $\mathrm{C} 4-7$ & $\mathrm{PO}_{100} \mathrm{H}_{30}+\mathrm{PO}_{100} \mathrm{H}_{30} \mathrm{~A}_{20}+\mathrm{CMC}_{20}-\mathrm{Hzd}+$ Dextran-Hzd & \\
\hline 49 & & $\mathrm{C} 4-8$ & $\mathrm{PO}_{100} \mathrm{H}_{30}+\mathrm{PO}_{100} \mathrm{H}_{30} \mathrm{~A}_{20}+\mathrm{CMC}_{20}-\mathrm{Hzd}+$ Chitosan-Hzd & \\
\hline 50 & & C4-9 & $\mathrm{PO}_{100} \mathrm{H}_{30}+\mathrm{PO}_{100} \mathrm{H}_{30} \mathrm{~A}_{20}+$ Dextran-Hzd + Chitosan-Hzd & \\
\hline 51 & & $\mathrm{C} 4-10$ & $\mathrm{PO}_{100} \mathrm{H}_{30}+\mathrm{CMC}_{20}-\mathrm{Hzd}+$ Dextran + Chitosan & \\
\hline 52 & & $\mathrm{C} 4-11$ & $\mathrm{PO}_{100} \mathrm{H}_{30} \mathrm{C}_{20}+\mathrm{PO}_{100} \mathrm{H}_{30} \mathrm{~A}_{20}+\mathrm{CMC}_{20}-\mathrm{Hzd}+$ Dextran-Hzd & \\
\hline 53 & & $\mathrm{C} 4-12$ & $\mathrm{PO}_{100} \mathrm{H}_{30} \mathrm{C}_{20}+\mathrm{PO}_{100} \mathrm{H}_{30} \mathrm{~A}_{20}+\mathrm{CMC}_{20}$-Hzd + Chitosan-Hzd & \\
\hline 54 & & $\mathrm{C} 4-13$ & $\mathrm{PO}_{100} \mathrm{H}_{30} \mathrm{C}_{20}+\mathrm{PO}_{100} \mathrm{H}_{30} \mathrm{~A}_{20}+$ Dextran-Hzd + Chitosan-Hzd & \\
\hline 55 & & $\mathrm{C} 4-14$ & $\mathrm{PO}_{100} \mathrm{H}_{30} \mathrm{C}_{20}+\mathrm{CMC}_{20}-\mathrm{Hzd}+$ Dextran-Hzd + Chitosan-Hzd & \\
\hline 56 & & $\mathrm{C} 4-15$ & $\mathrm{PO}_{100} \mathrm{H}_{30} \mathrm{~A}_{20}+\mathrm{CMC}_{20}-\mathrm{Hzd}+$ Dextran-Hzd + Chitosan-Hzd & \\
\hline 57 & \multirow{6}{*}{$C_{6}^{5}$} & $\mathrm{C} 5-1$ & $\mathrm{PO}_{100} \mathrm{H}_{30}+\mathrm{PO}_{100} \mathrm{H}_{30} \mathrm{C}_{20}+\mathrm{CMC}_{20}-\mathrm{Hzd}+\mathrm{CMC}_{20}-\mathrm{Hzd}+$ Dextran-Hzd & \multirow{6}{*}{$12 \mu \mathrm{L}$} \\
\hline 58 & & $\mathrm{C} 5-2$ & $\mathrm{PO}_{100} \mathrm{H}_{30}+\mathrm{PO}_{100} \mathrm{H}_{30} \mathrm{C}_{20}+\mathrm{CMC}_{20}-\mathrm{Hzd}+\mathrm{CMC}_{20}-\mathrm{Hzd}+$ Chitosan-Hzd & \\
\hline 59 & & $\mathrm{C} 5-3$ & $\mathrm{PO}_{100} \mathrm{H}_{30}+\mathrm{PO}_{100} \mathrm{H}_{30} \mathrm{C}_{20}+\mathrm{CMC}_{20}-\mathrm{Hzd}+$ Dextran-Hzd + Chitosan-Hzd & \\
\hline 60 & & $\mathrm{C} 5-4$ & $\mathrm{PO}_{100} \mathrm{H}_{30}+\mathrm{PO}_{100} \mathrm{H}_{30} \mathrm{C}_{20}+\mathrm{CMC}_{20}-\mathrm{Hzd}+$ Dextran-Hzd + Chitosan-Hzd & \\
\hline 61 & & $\mathrm{C} 5-5$ & $\mathrm{PO}_{100} \mathrm{H}_{30}+\mathrm{PO}_{100} \mathrm{H}_{30} \mathrm{~A}_{20}+\mathrm{CMC}_{20}-\mathrm{Hzd}+$ Dextran-Hzd + Chitosan-Hzd & \\
\hline 62 & & C5-6 & $\mathrm{PO}_{100} \mathrm{H}_{30} \mathrm{C}_{20}+\mathrm{PO}_{100} \mathrm{H}_{30} \mathrm{~A}_{20}+\mathrm{CMC}_{20}-\mathrm{Hzd}+$ Dextran-Hzd + Chitosan-Hzd & \\
\hline 63 & $C_{6}^{6}$ & C6-1 & $\begin{array}{l}\mathrm{PO}_{100} \mathrm{H}_{30}+\mathrm{PO}_{100} \mathrm{H}_{30} \mathrm{C}_{20}+\mathrm{PO}_{100} \mathrm{H}_{30} \mathrm{~A}_{20}+\mathrm{CMC}_{20}-\mathrm{Hzd}+\text { Dextran-Hzd } \\
+ \text { Chitosan-Hzd }\end{array}$ & $10 \mu \mathrm{L}$ \\
\hline
\end{tabular}

* The combinations of hydrazide polymers listed above were each mixed with a single aldehyde precursor polymer $\left(\mathrm{PO}_{100} \mathrm{~A}_{30}, 60 \mu \mathrm{L}\right)$ to facilitate gelation. The total volume of each gel was 120 $\mu \mathrm{L}$. CMC-Hzd was added at a concentration of $20 \mathrm{mg} / \mathrm{mL}$ 


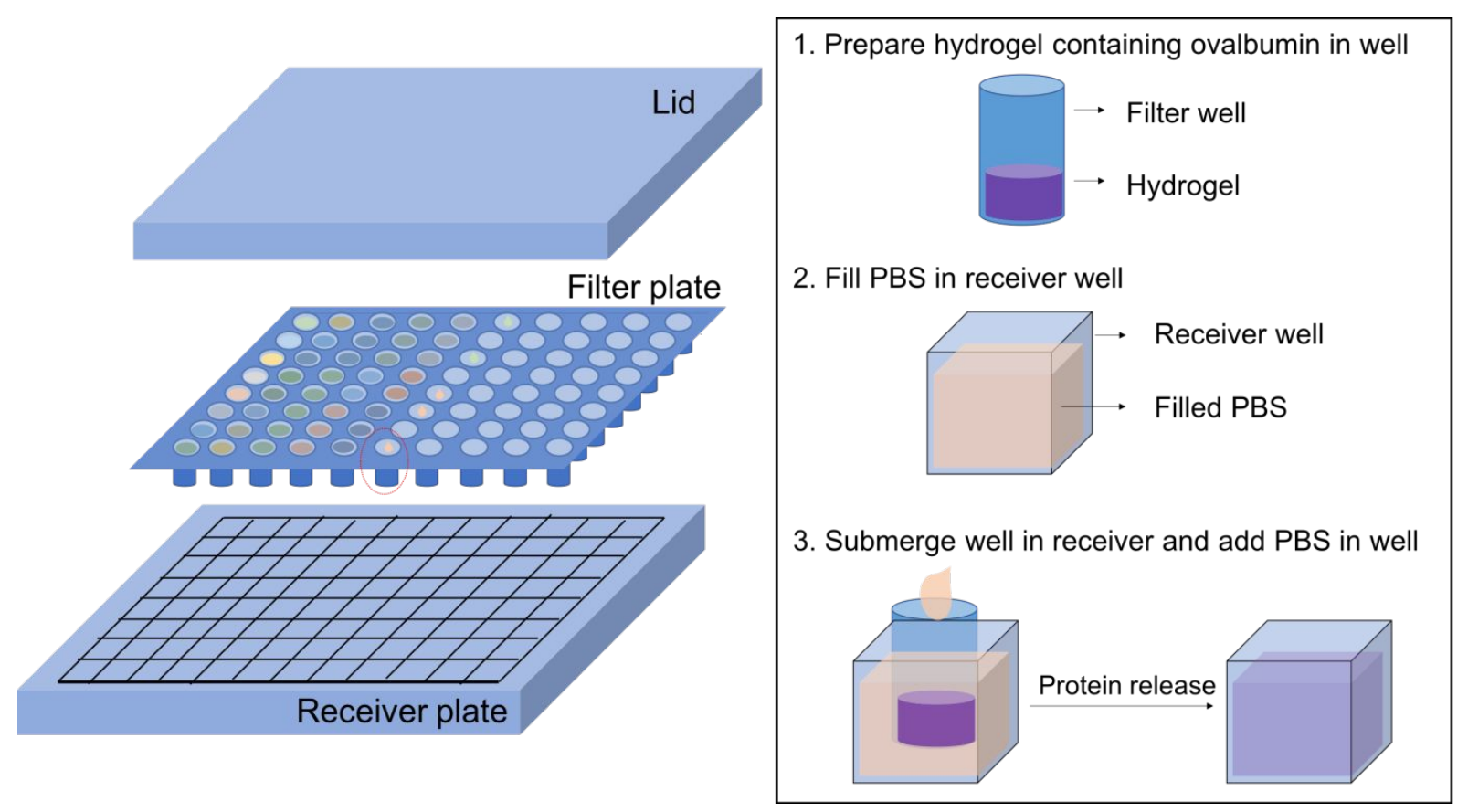

Figure S1. Schematic of drug release set-up in 96 well-plate using high-throughput measurement.
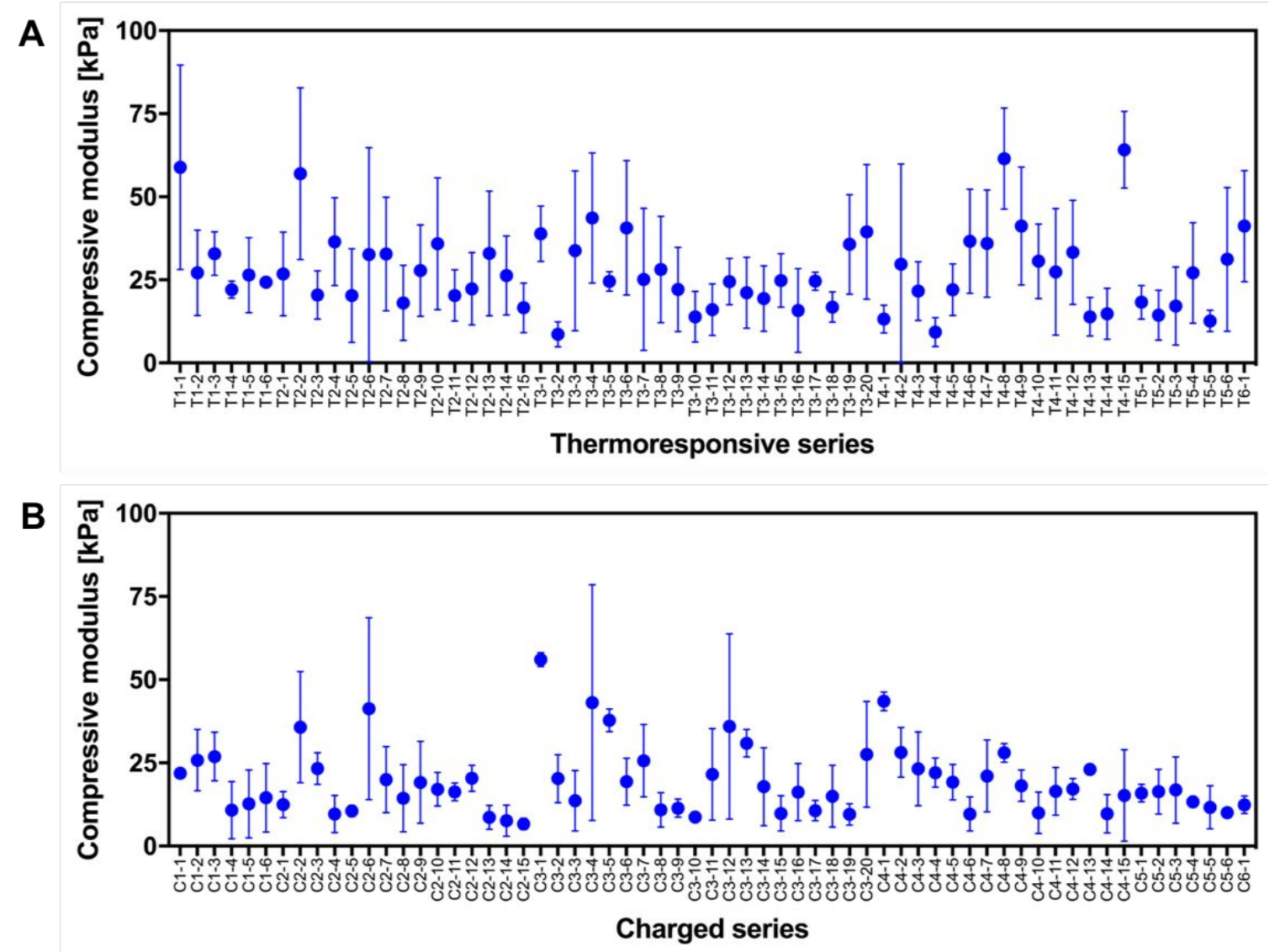

Figure S2. Compression modulus of each hydrogel before swelling. ( $\mathrm{t}=0)$ 
Cytocompatibility testing. The in vitro cytocompatibility of all the polymer precursors was assessed using NIH $3 \mathrm{~T} 3$ mouse fibroblasts. Cells were cultured in DMEM containing 10\% FBS and $1 \%$ PS at $37{ }^{\circ} \mathrm{C}$ with $5 \% \mathrm{CO}_{2}$ to $\sim 80 \%$ confluence before use. 10,000 cells were plated in each well of 96 well-plate and incubated overnight in $100 \mu \mathrm{L}$ DMEM medium. Following, the DMEM was aspirated and replaced with polymer precursor solutions at concentrations of $1 \mathrm{mg} / \mathrm{mL}$ and $2 \mathrm{mg} / \mathrm{mL}$ in the same DMEM media and incubated for an additional $24 \mathrm{~h}$. Following, cell viability was assessed using the PrestoBlue assay following the standard protocol, with absorbances measured using a microplate reader (Infinite M200 Pro) at wavelength of $570 \mathrm{~nm}$. Cell viabilities were calculated by comparing the measured PrestoBlue signal in the presence of the polymers to cell-only and media-only controls. Error bars represent the standard deviation of four independent measurements $(\mathrm{n}=4)$.

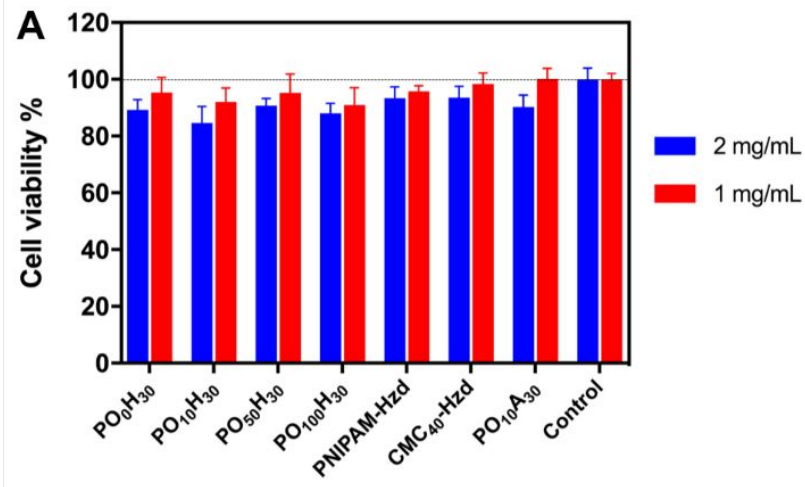

Polymer precursors

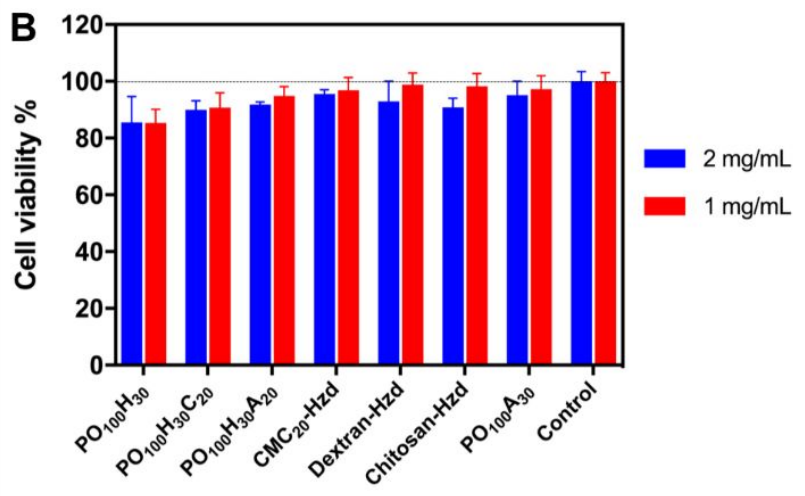

Polymer precursors

Figure S3. 3T3 mouse fibroblast cell viability (relative to cell-only control) of polymer precursors used to produce (A) the thermoresponsive vs. non-thermoresponsive combinatorial hydrogels and (B) the charged vs. neutral combinatorial hydrogels $(n=4)$. Note that due to the rapid gelation of the precursor polymers $(<1$ minute) coupled with the slow degradation of the materials (days to months at physiological $\mathrm{pH}$ ), the effective concentration of each polymer to which cells are exposed is much lower than the concentrations injected, consistent with positive tissue compatibility tests with many of these precursor polymers in vivo. 

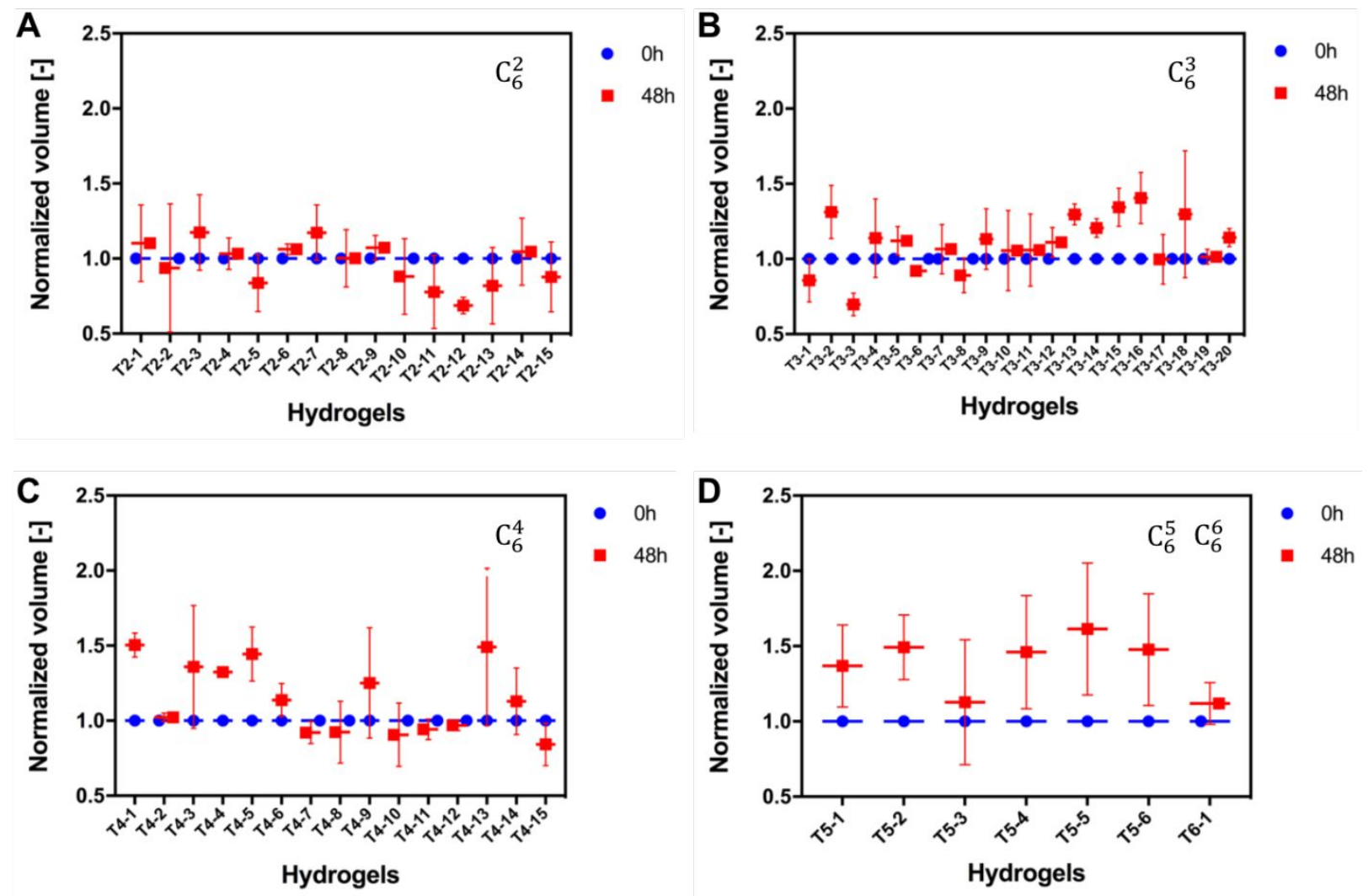

Figure S4. Volume-based swelling ratios of combinatorial thermoresponsive vs. nonthermoresponsive hydrogels before and after swelling for 48 hours in $10 \mathrm{mM}$ PBS at room temperature $\left(22^{\circ} \mathrm{C}\right)(\mathrm{n}=4)$. The gel composition corresponding to each label along the $\mathrm{x}$-axis can be referenced in Table $\mathrm{S} 1$. 



Figure S5. Volume-based swelling ratios of combinatorial charged vs. neutral hydrogels before and after swelling for 48 hours in $10 \mathrm{mM}$ PBS at room temperature $\left(22{ }^{\circ} \mathrm{C}\right)(\mathrm{n}=4)$. The gel composition corresponding to each label along the $\mathrm{x}$-axis can be referenced in Table S2.

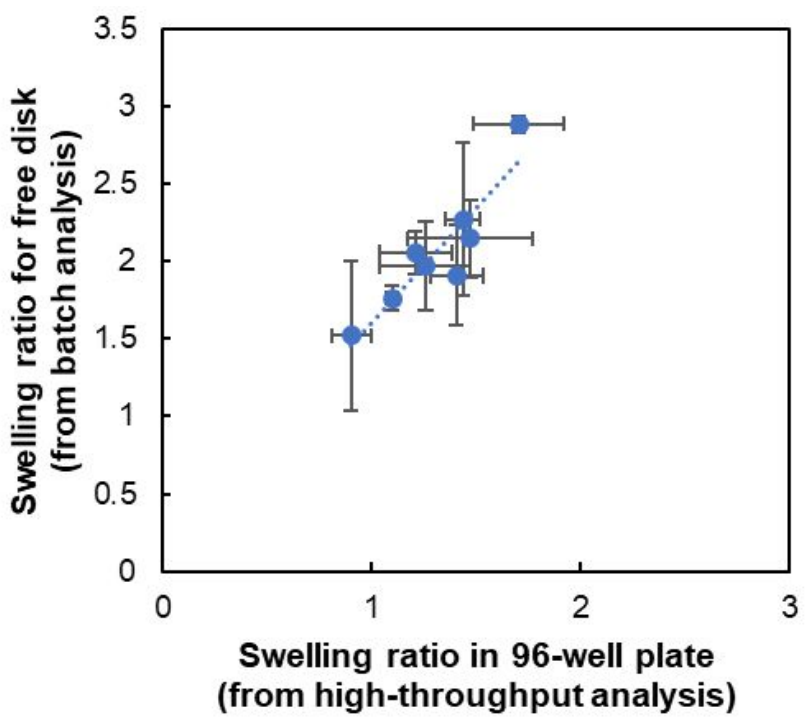

Figure S6. Comparison of the equilibrium swelling ratio measured in the 96-well plate high throughput assay (constrained swelling) versus for a free disk (unconstrained swelling). $\mathrm{R}^{2}=$ 0.84), showing the predictive nature of the high-throughput measurements. 

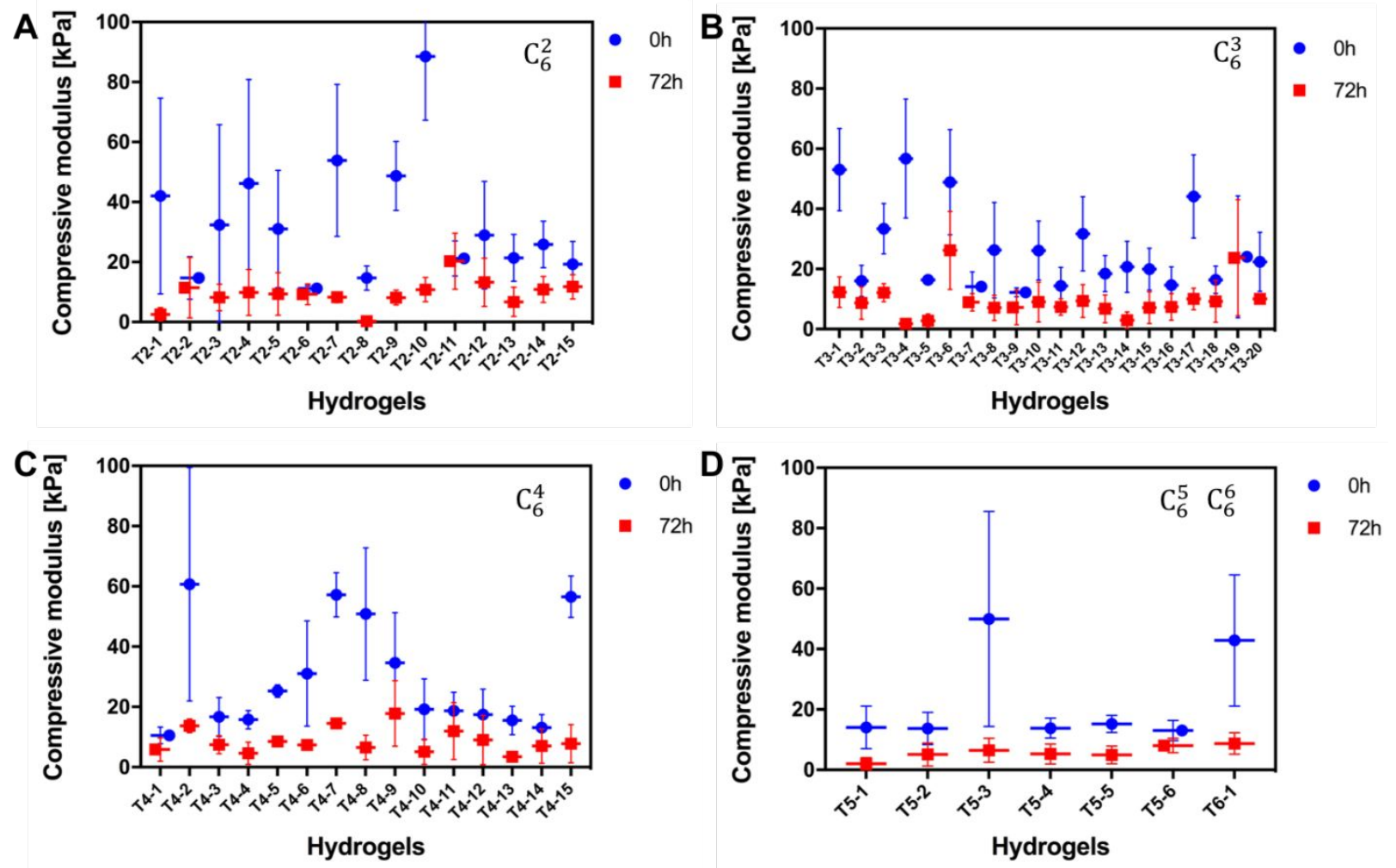

Figure S7. Compressive moduli of combinatorial thermoresponsive vs. non-thermoresponsive hydrogels immediately after fabrication (blue, corresponding to the reported compressive modulus results) and after 72 hours immersed in $100 \mathrm{mM} \mathrm{HCl}$ (red, corresponding to the reported degradation results) at room temperature $\left(2{ }^{\circ} \mathrm{C}\right)(\mathrm{n}=4)$. The gel composition corresponding to each label along the $\mathrm{x}$-axis can be referenced in Table S1. 

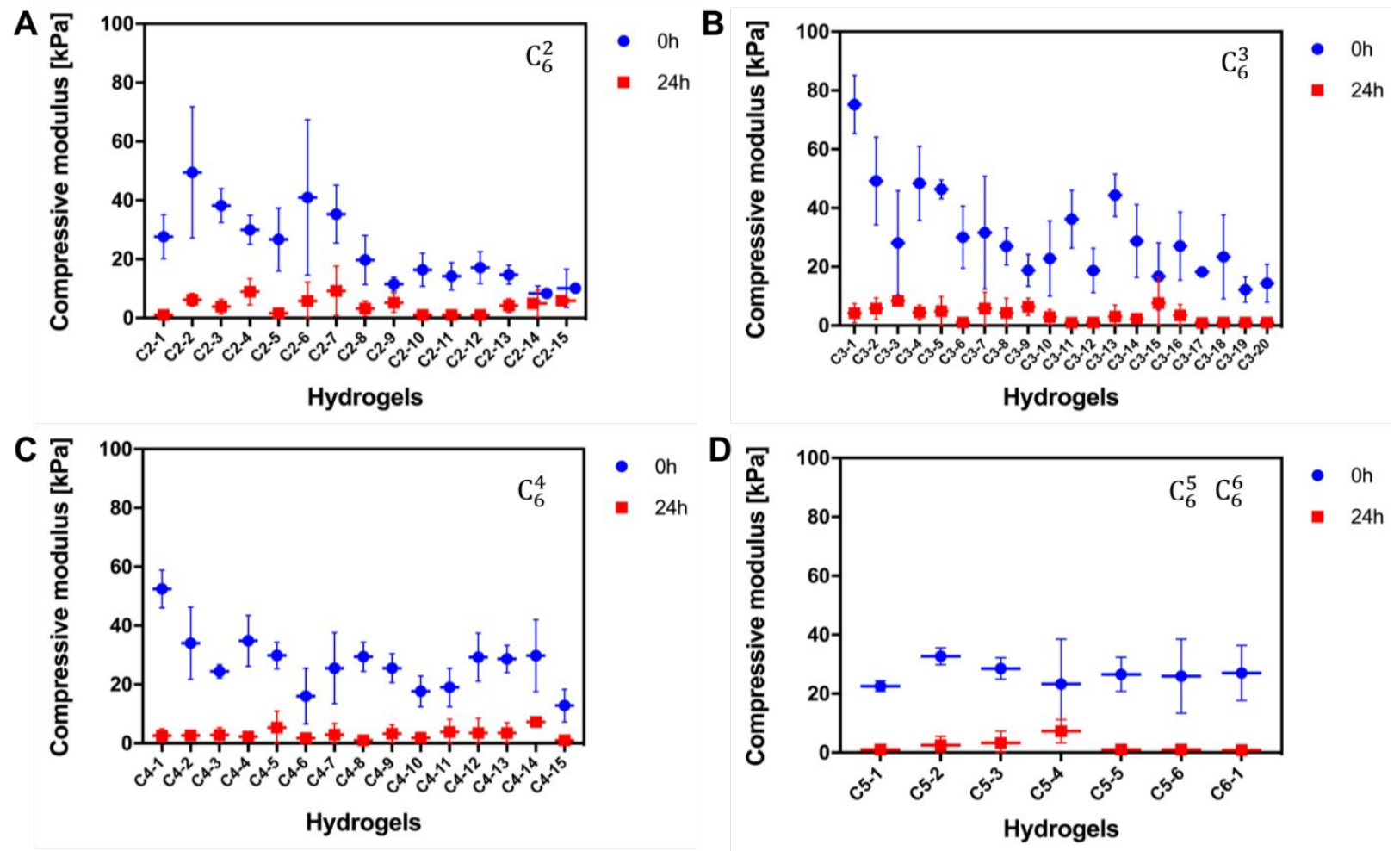

Figure S8. Compressive moduli of combinatorial charged vs. neutral hydrogels immediately after fabrication (blue, corresponding to the reported compressive modulus results) and after 72 hours immersed in $100 \mathrm{mM} \mathrm{HCl}$ (red, corresponding to the reported degradation results) at room temperature $\left(22^{\circ} \mathrm{C}\right)(\mathrm{n}=4)$. The gel composition corresponding to each label along the $\mathrm{x}$-axis can be referenced in Table S2. 

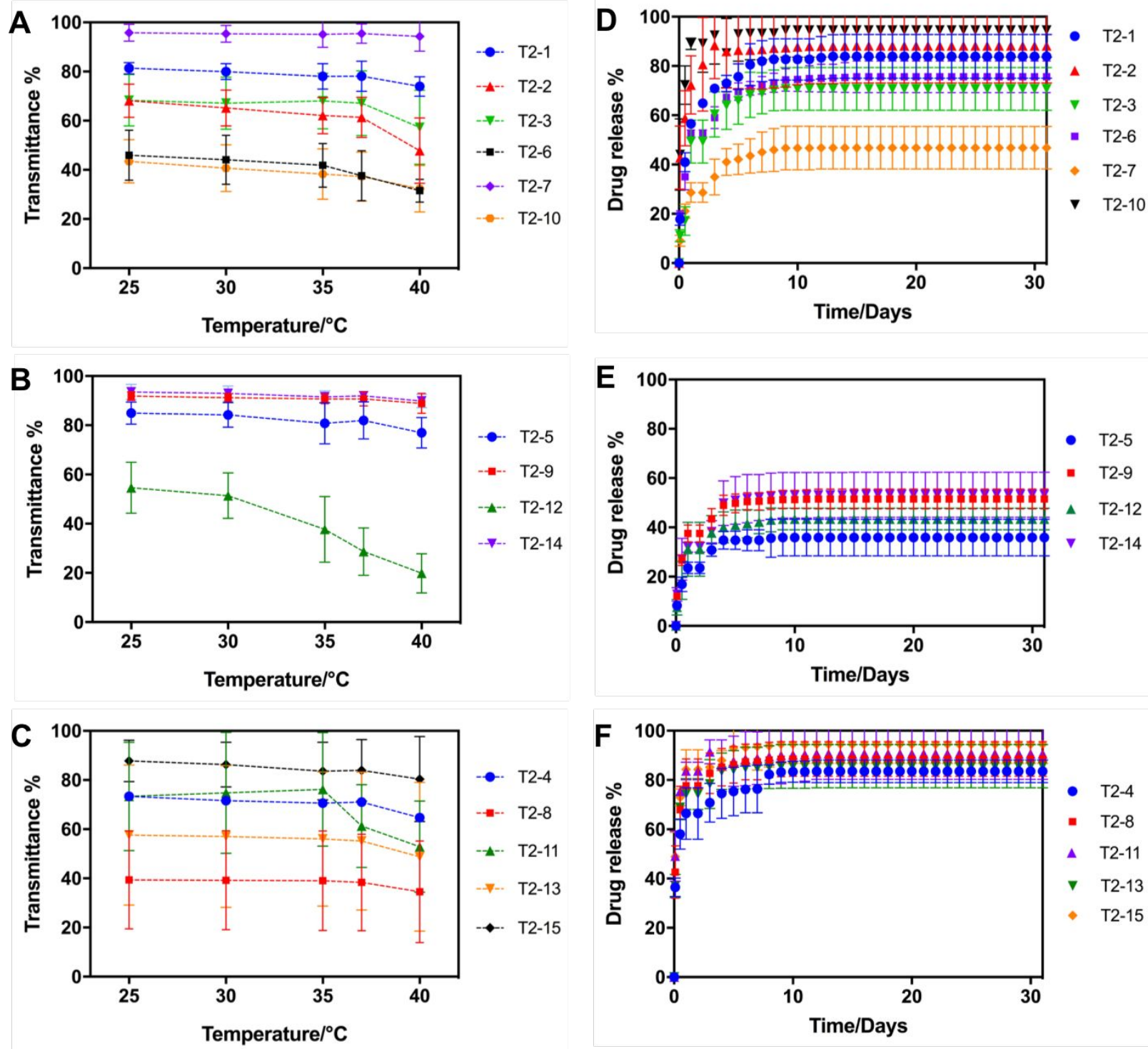

Figure S9. Transmittance (A-C) and release kinetics of ovalbumin (D-F) from thermoresponsive vs. non-thermoresponsive combinatorial hydrogels $(n=4)$ containing two different hydrazide precursors including (A,D) $\mathrm{PO}_{\mathrm{x}} \mathrm{H}_{\mathrm{y}}$ only, $(\mathrm{B}, \mathrm{E}) \mathrm{PO}_{\mathrm{x}} \mathrm{H}_{\mathrm{y}}+$ PNIPAM-Hzd composition and $(\mathrm{C}, \mathrm{F})$ $\mathrm{PO}_{\mathrm{x}} \mathrm{H}_{\mathrm{y}}+\mathrm{CMC}_{40}-\mathrm{Hzd}$ composition. The gel composition corresponding to each label in the legend can be referenced in Table S1. Protein release was measured in $10 \mathrm{mM}$ PBS at $37^{\circ} \mathrm{C}$. 

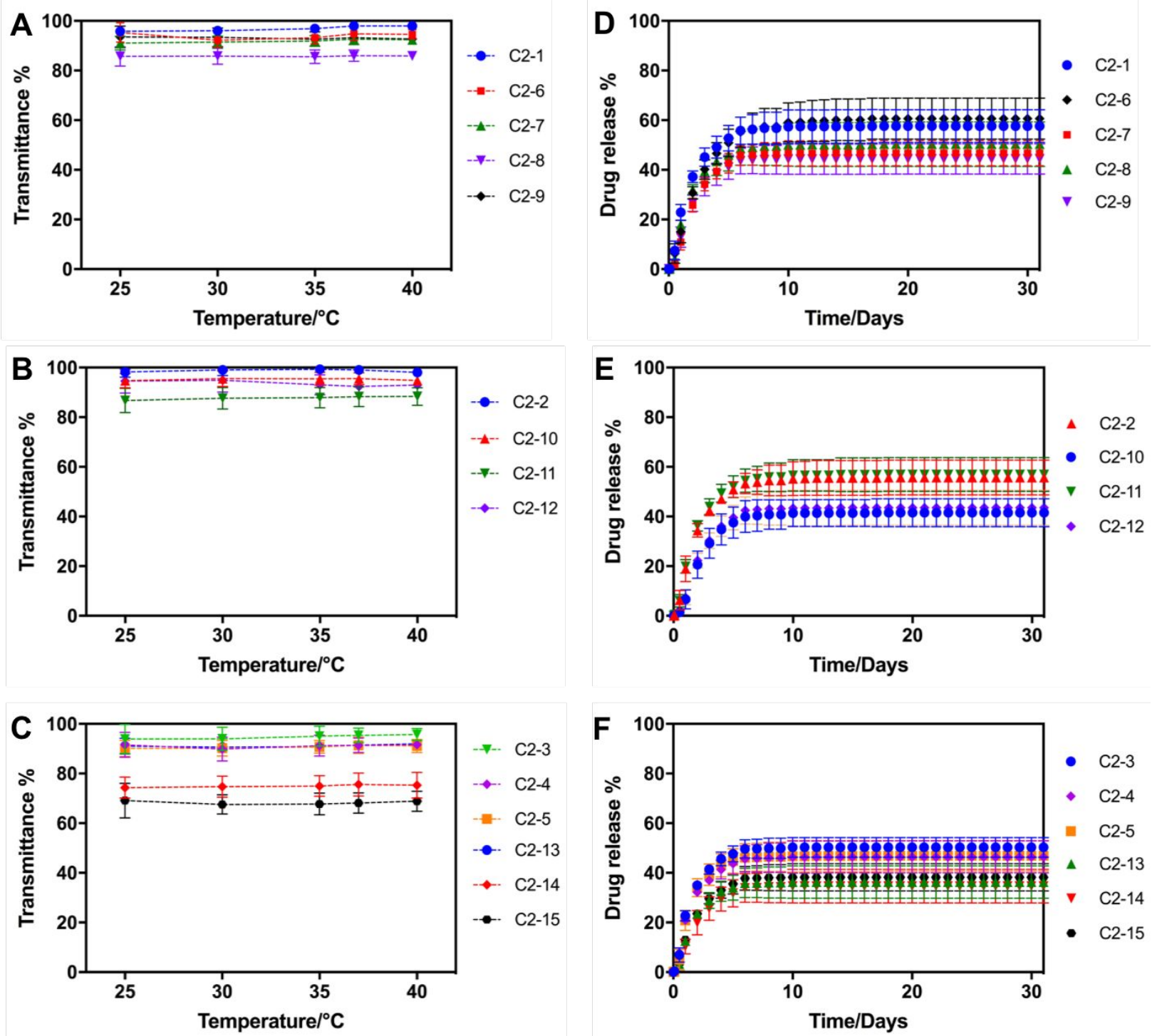

Figure S10. Transmittance (A-C) and release kinetics of ovalbumin (D-F) from charged vs. neutral combinatorial hydrogels $(n=4)$ containing two different hydrazide precursors including $(A, D)$ $\mathrm{PO}_{100} \mathrm{H}_{30} \mathrm{C}_{20}$, (B,E) $\mathrm{PO}_{100} \mathrm{H}_{30} \mathrm{~A}_{20}$ and $(\mathrm{C}, \mathrm{F}) \mathrm{PO}_{100} \mathrm{H}_{30}$. The gel composition corresponding to each label in the legend can be referenced in Table S2. Protein release was measured in $10 \mathrm{mM}$ PBS at $37^{\circ} \mathrm{C}$. 

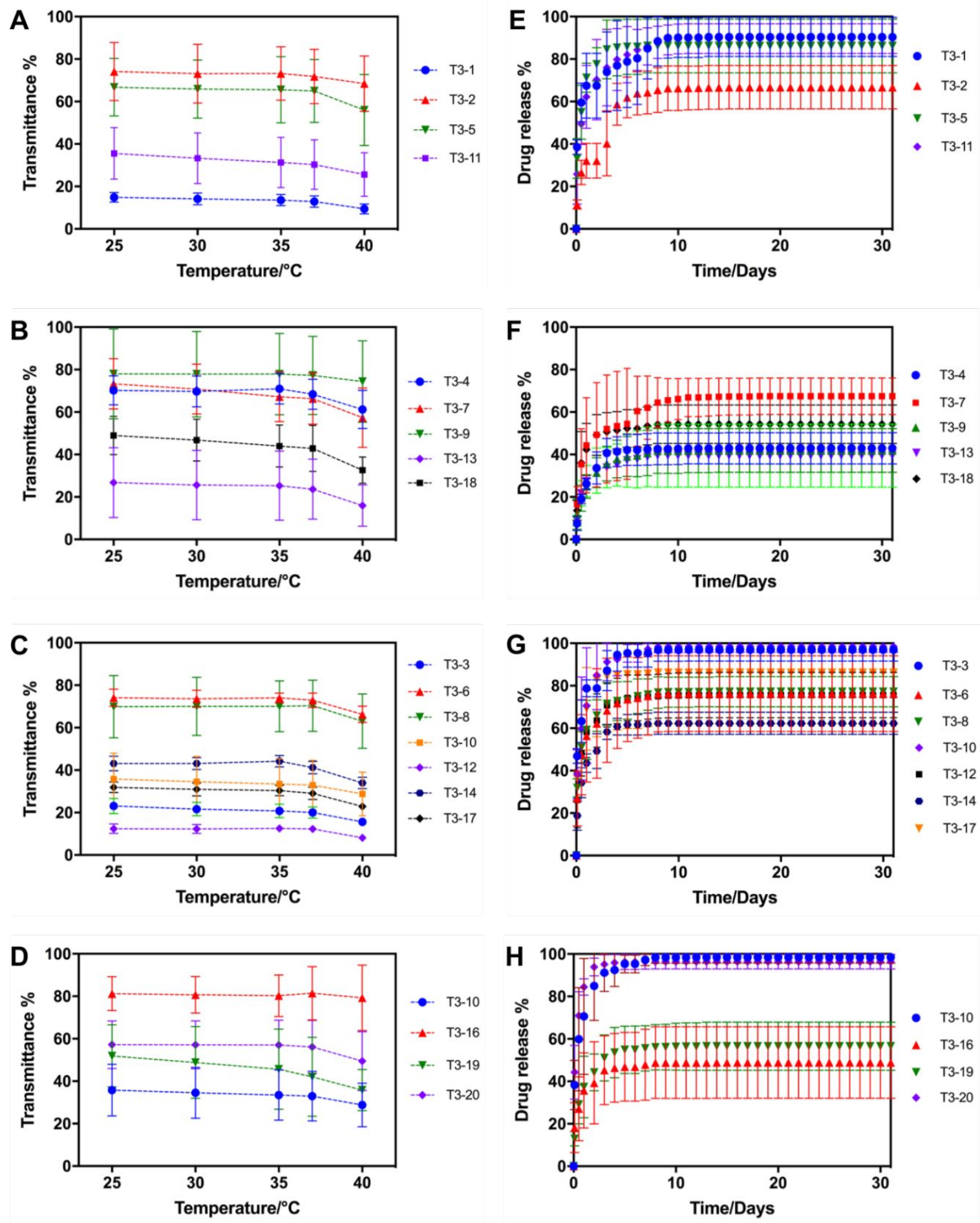

Figure S11. Transmittance (A-D) and release kinetics of ovalbumin (E-H) from thermoresponsive vs. non-thermoresponsive combinatorial hydrogels $(n=4)$ containing three different hydrazide precursors such as (A,E) $\mathrm{PO}_{\mathrm{x}} \mathrm{H}_{\mathrm{y}}$ only; $(\mathrm{B}, \mathrm{F}) \mathrm{PO}_{\mathrm{x}} \mathrm{H}_{\mathrm{y}}+\mathrm{CMC}_{40}-\mathrm{Hzd} ;(\mathrm{C}, \mathrm{G}) \mathrm{PO}_{\mathrm{x}} \mathrm{H}_{\mathrm{y}}+\mathrm{PNIPAM}-\mathrm{Hzd}$; and $(\mathrm{D}, \mathrm{H}) \mathrm{PO}_{\mathrm{x}} \mathrm{H}_{\mathrm{y}}+\mathrm{CMC}_{40}$-Hzd + PNIPAM-Hzd. The gel composition corresponding to each label in the legend can be referenced in Table S1. Protein release was measured in $10 \mathrm{mM}$ PBS at $37^{\circ} \mathrm{C}$. 

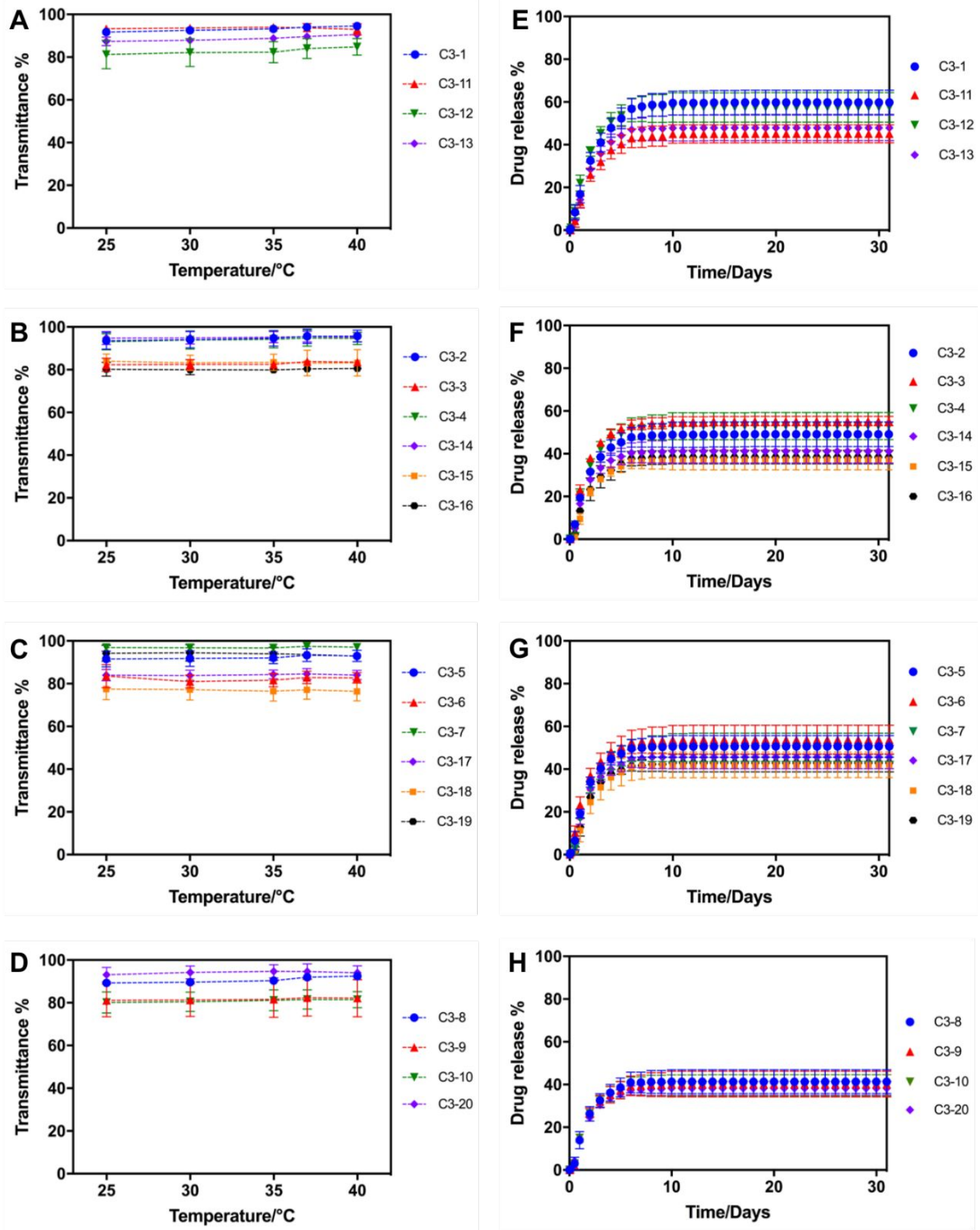

Figure S12. Transmittance (A-D) and release kinetics of ovalbumin (E-H) from charged vs. neutral combinatorial hydrogels $(\mathrm{n}=4)$ containing three different hydrazide precursors such as $(\mathrm{A}, \mathrm{E})$ both synthetic charged polymers $\mathrm{PO}_{100} \mathrm{H}_{30} \mathrm{C}_{20}$ and $\mathrm{PO}_{100} \mathrm{H}_{30} \mathrm{~A}_{20}$ included; (B,F) only one synthetic charged polymer $\mathrm{PO}_{100} \mathrm{H}_{30} \mathrm{C}_{20}$ included; $(\mathrm{C}, \mathrm{G})$ only one synthetic charged polymer $\mathrm{PO}_{100} \mathrm{H}_{30} \mathrm{~A}_{20}$ included; and $(\mathrm{D}, \mathrm{H})$ neither $\mathrm{PO}_{100} \mathrm{H}_{30} \mathrm{C}_{20}$ or $\mathrm{PO}_{100} \mathrm{H}_{30} \mathrm{~A}_{20}$ included. The gel composition corresponding to each label in the legend can be referenced in Table S2. Protein release was measured in $10 \mathrm{mM}$ PBS at $37^{\circ} \mathrm{C}$. 

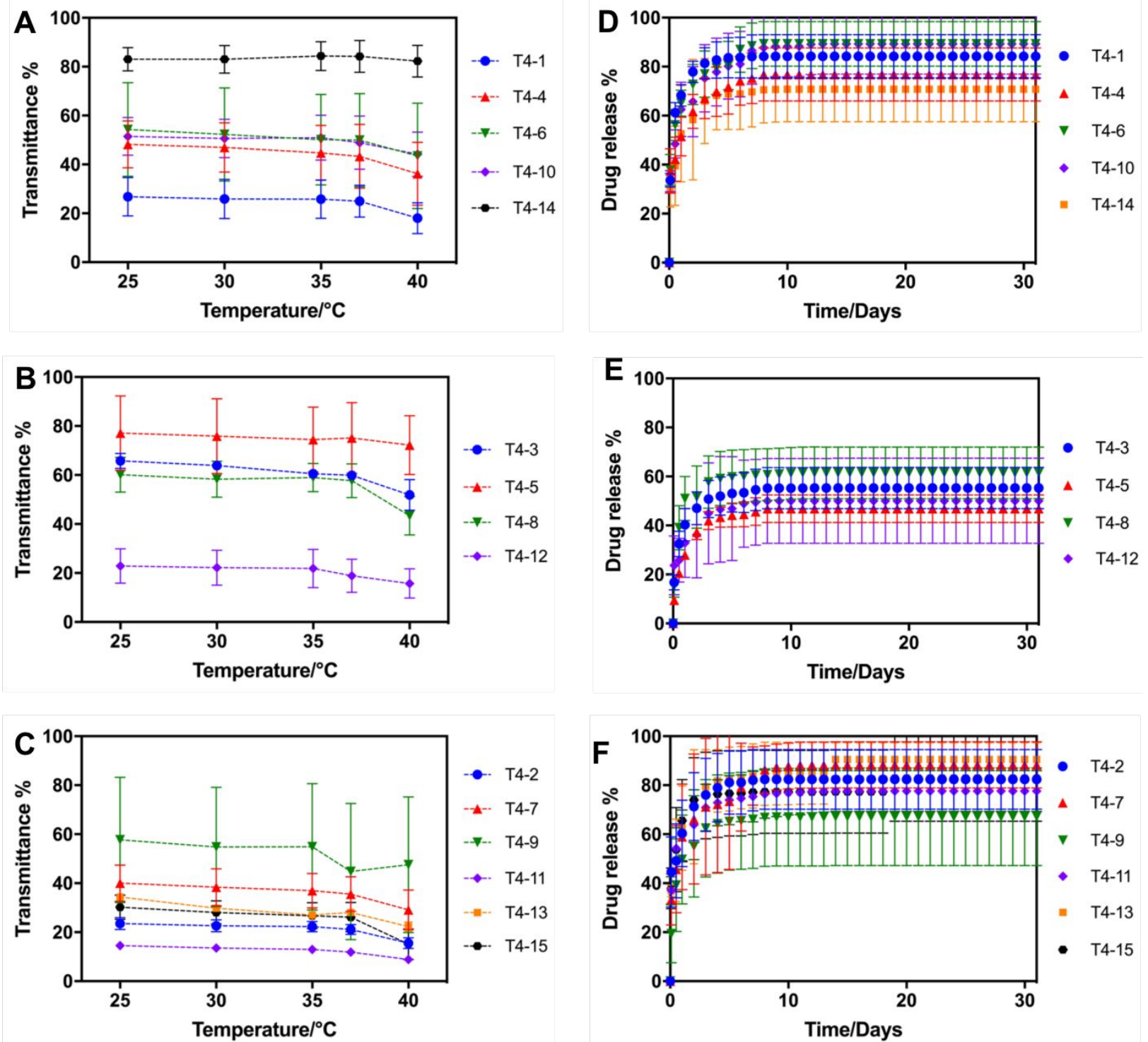

Figure S13. Transmittance (A-C) and release kinetics of ovalbumin (D-F) from thermoresponsive vs. non-thermoresponsive combinatorial hydrogels $(n=4)$ containing four different hydrazide precursors including (A,D) $\mathrm{PO}_{\mathrm{x}} \mathrm{H}_{\mathrm{y}},(\mathrm{B}, \mathrm{E}) \mathrm{PO}_{\mathrm{x}} \mathrm{H}_{\mathrm{y}}+\mathrm{CMC}-\mathrm{Hzd}$; and $(\mathrm{C}, \mathrm{F})$ both $\mathrm{PO}_{0} \mathrm{H}_{30}$ and PNIPAM-Hzd included. The gel composition corresponding to each label in the legend can be referenced in Table S1. Protein release was measured in $10 \mathrm{mM}$ PBS at $37{ }^{\circ} \mathrm{C}$. 

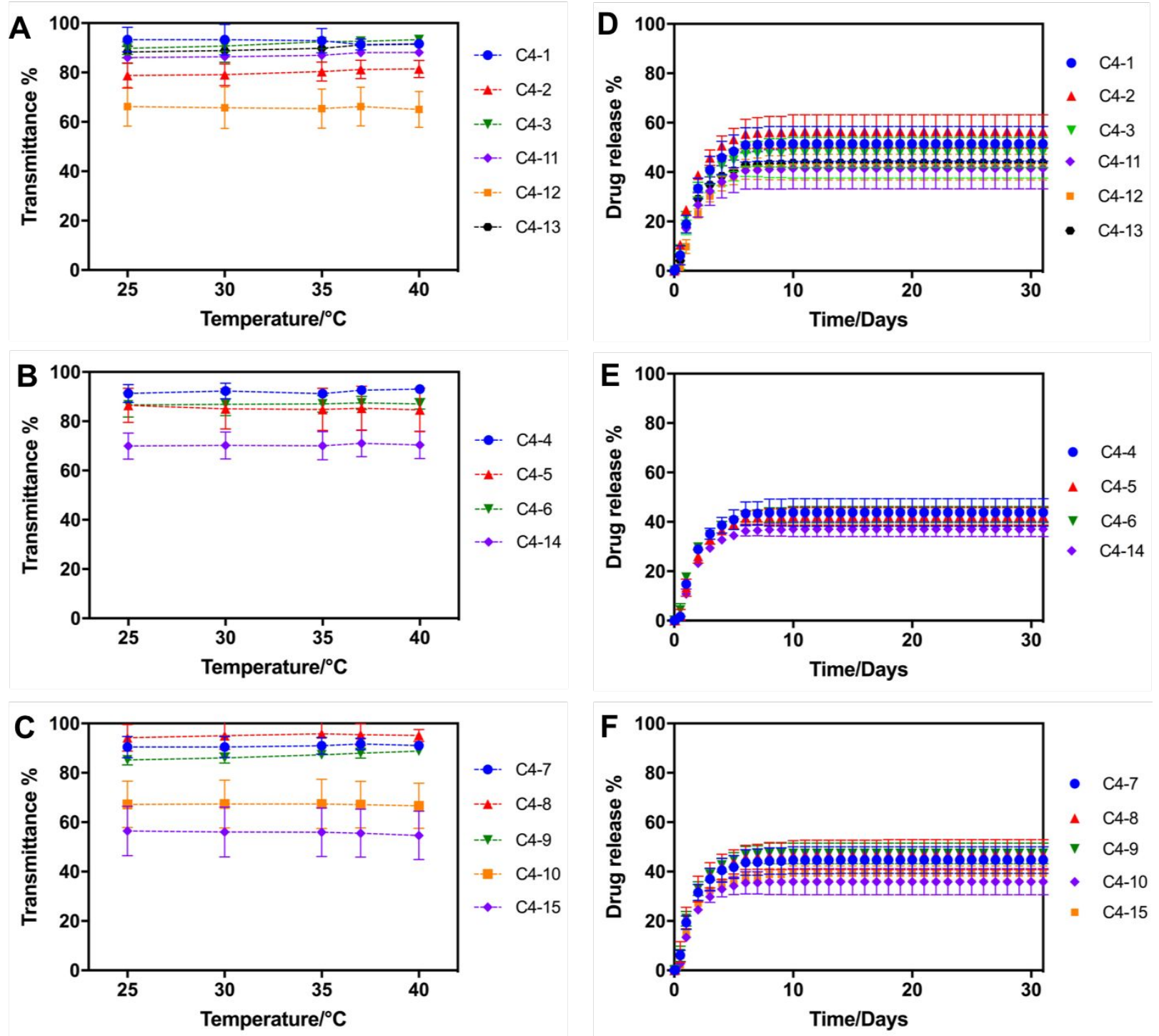

Figure S14. Transmittance (A-C) and release kinetics of ovalbumin (D-F) from charged vs. neutral combinatorial hydrogels $(n=4)$ containing four different hydrazide precursors including $(A, D)$ both synthetic charged polymers $\mathrm{PO}_{100} \mathrm{H}_{30} \mathrm{C}_{20}$ and $\mathrm{PO}_{100} \mathrm{H}_{30} \mathrm{~A}_{20}$ included; $(\mathrm{B}, \mathrm{E})$ only one synthetic charged polymer $\mathrm{PO}_{100} \mathrm{H}_{30} \mathrm{C}_{20}$ included; $(\mathrm{C}, \mathrm{F})$ no $\mathrm{PO}_{100} \mathrm{H}_{30} \mathrm{C}_{20}$. The gel composition corresponding to each label in the legend can be referenced in Table S2. Protein release was measured in $10 \mathrm{mM}$ PBS at $37^{\circ} \mathrm{C}$. 

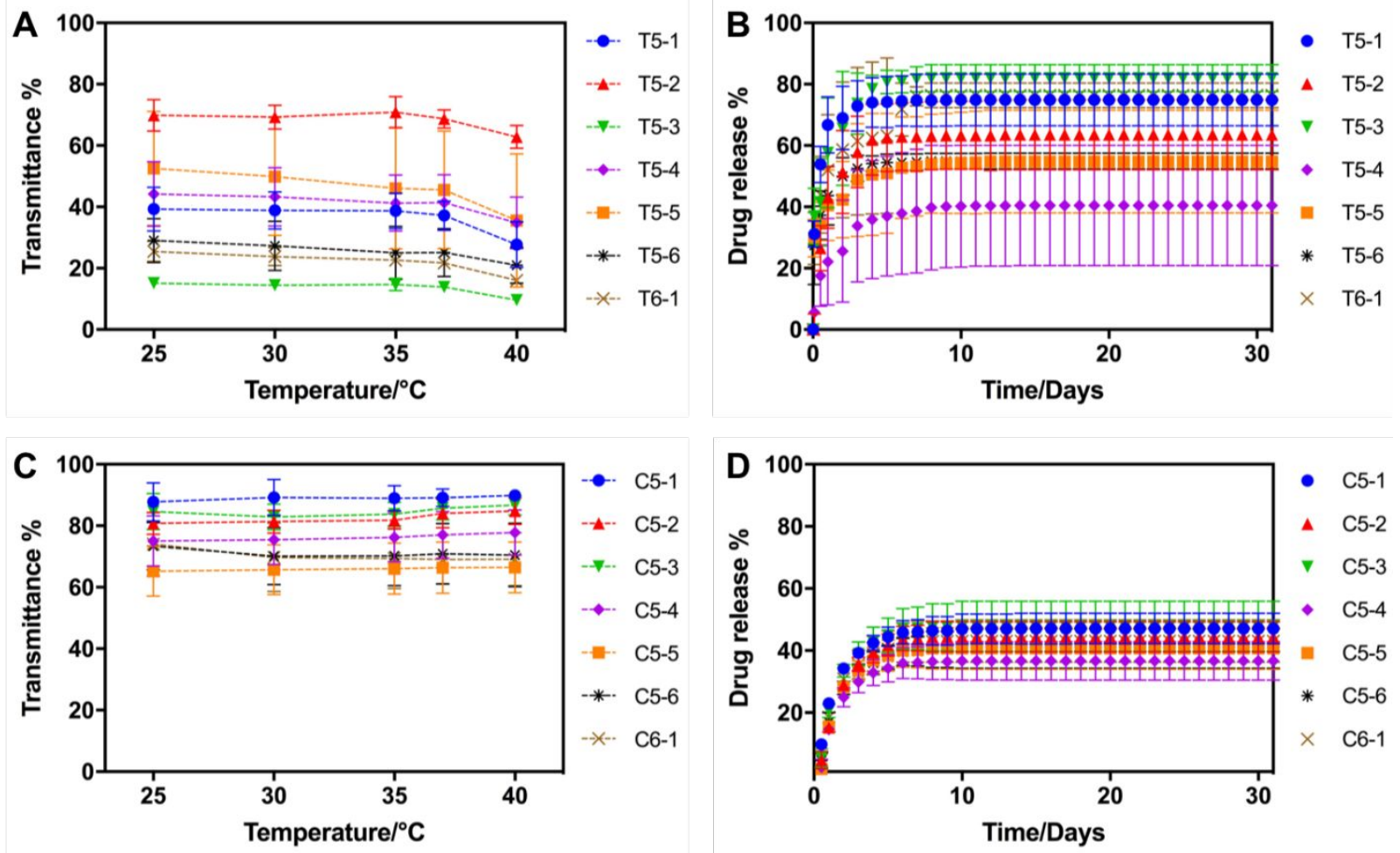

Figure S15. Transmittance (A, C) and release kinetics of ovalbumin (B, D) from thermoresponsive vs. non-thermoresponsive (A-B) and charged vs. neutral (C-D) combinatorial hydrogels $(n=4)$ containing five/six different hydrazide precursors. The gel composition corresponding to each label in the legend can be referenced in Table S1 and S2. Protein release was measured in $10 \mathrm{mM}$ PBS at $37^{\circ} \mathrm{C}$. 


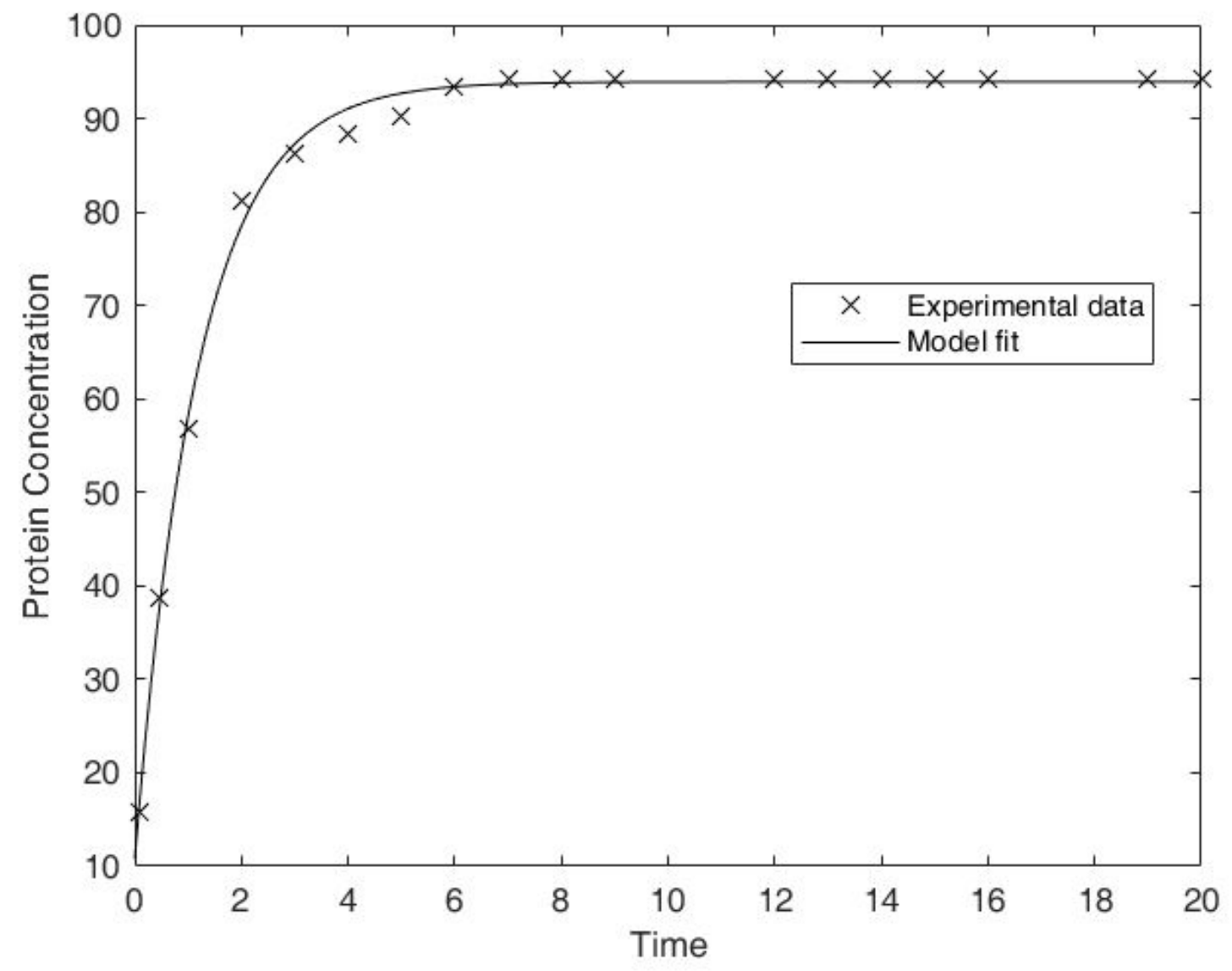

Figure S16. Representative model fit of high-throughput ovalbumin release kinetics using the modified first order model $\hat{y}(t)=y_{f}+\left(y_{0}-y_{f}\right) e^{-t / \tau}$. 




Figure S17. Root mean squared errors of modified first-order fits to $\hat{y}(t)=y_{f}+\left(y_{0}-y_{f}\right) e^{-t / \tau}$ to ovalbumin release kinetics data from both thermoresponsive (T series) and charged (C series) high-throughput hydrogels. The results suggest good model fits for almost all assessed hydrogels using this modified first-order model, allowing the kinetic parameters acquired from the model to be used in subsequent iterative model optimization. The dashed line in this figure represents our threshold for inclusion in subsequent latent variable modeling, selected based on visual evaluation of the resulting model fit. 


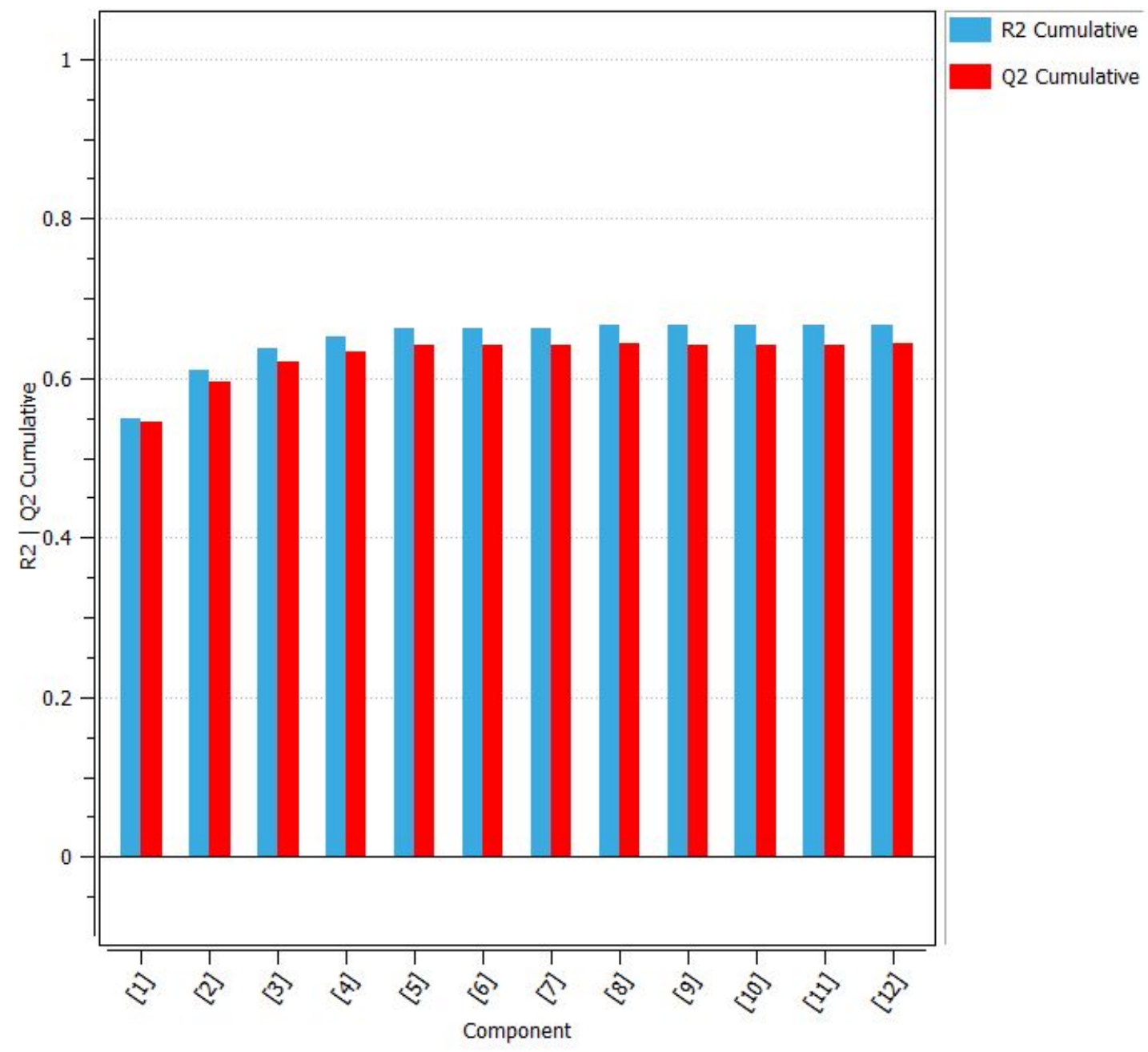

Figure S18. $\mathrm{R}^{2}$ (percentage variation explained) and $\mathrm{Q}^{2}$ (variation explained during cross validation with 7 groups) for the multivariate model constructed for predicting hydrogel transparency and drug release kinetics parameters. 


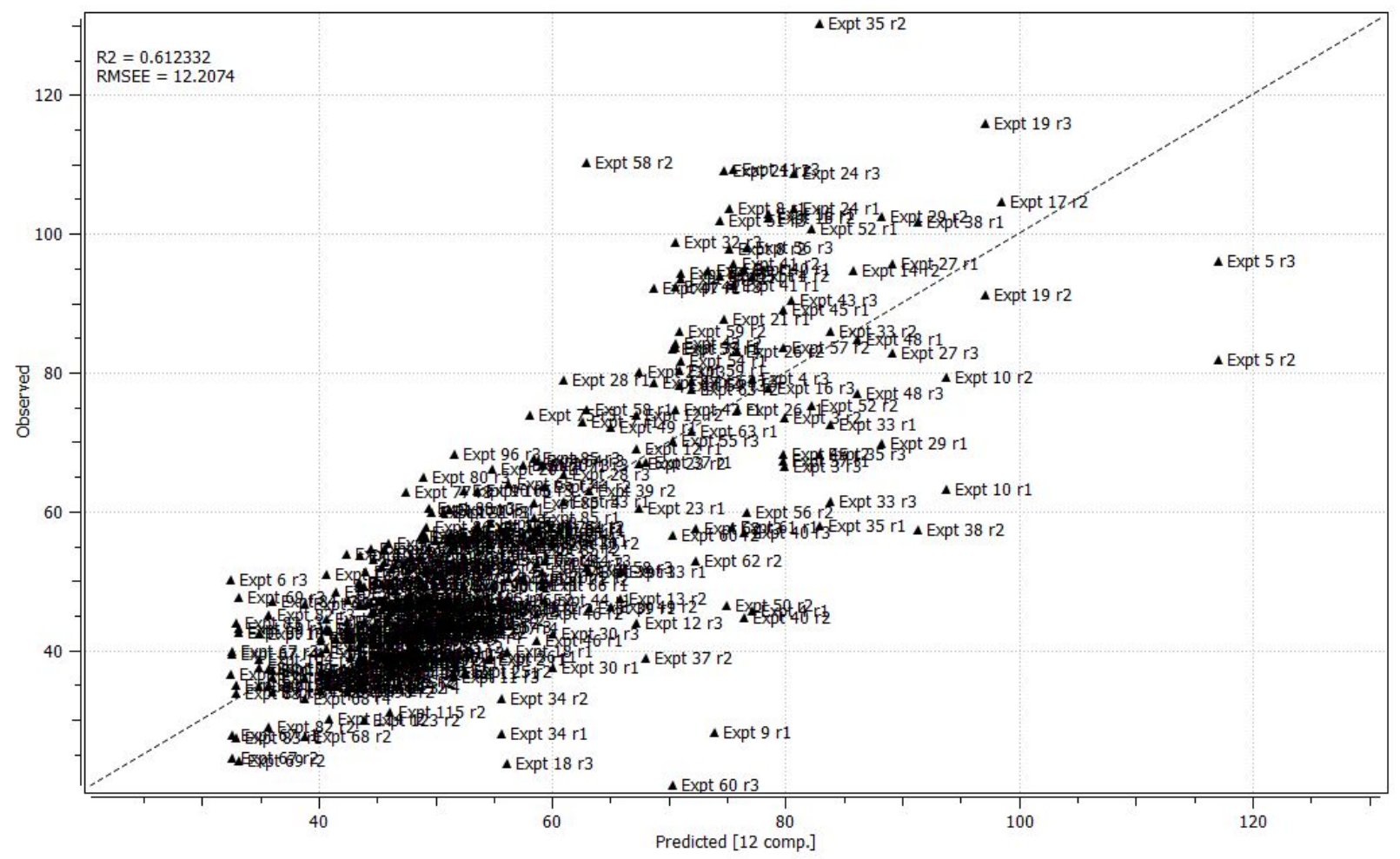

Figure S19. Observed (y-axis) versus predicted (x-axis) values for the plateau release parameter $y_{f}$ based on the 12-component PLS model assembled for ovalbumin release from high-throughput fabricated hydrogels 


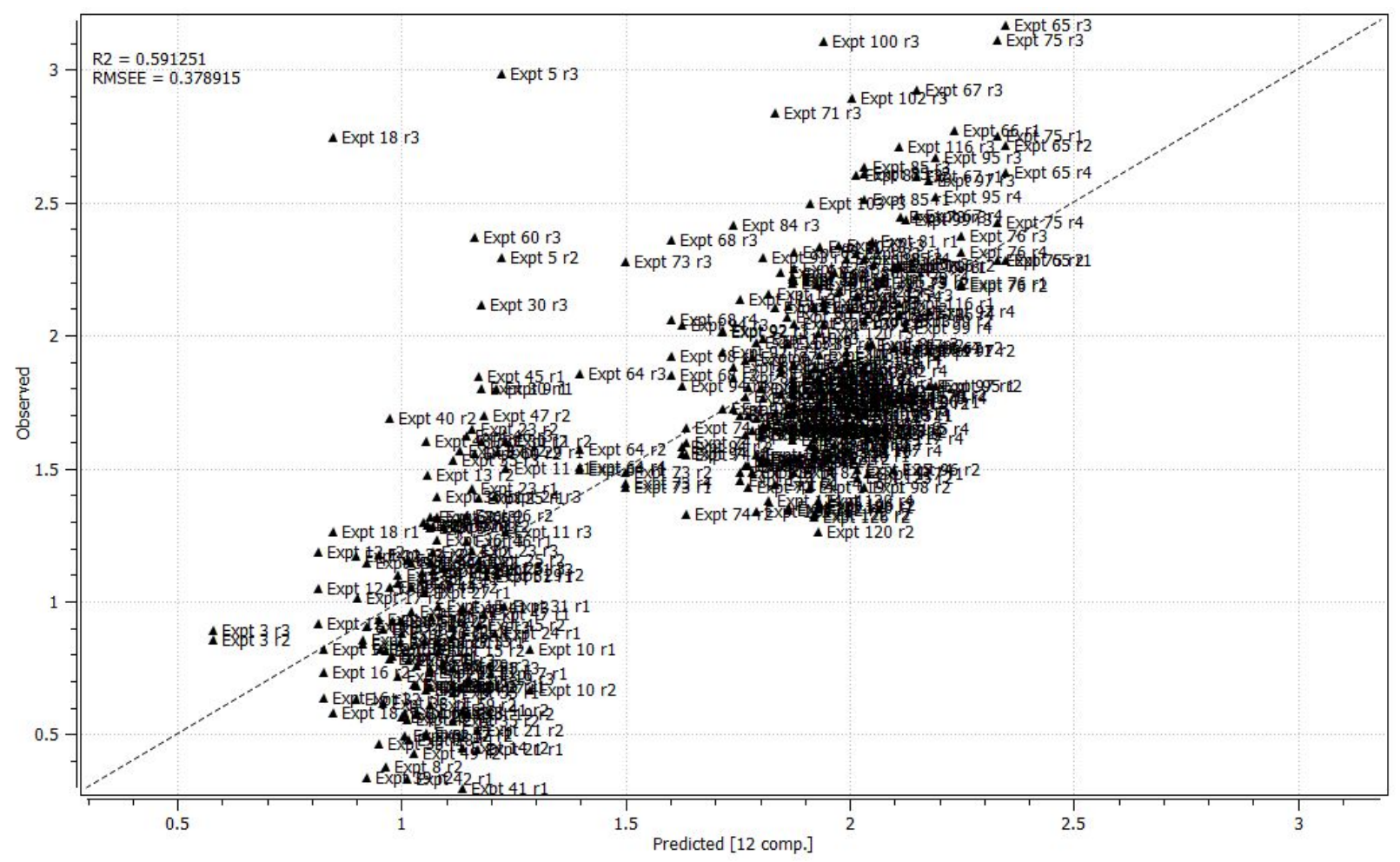

Figure S20. Observed (y-axis) versus predicted (x-axis) values for the first order kinetic release parameter $\tau$ based on the 12-component PLS model assembled for ovalbumin release from highthroughput fabricated hydrogels 


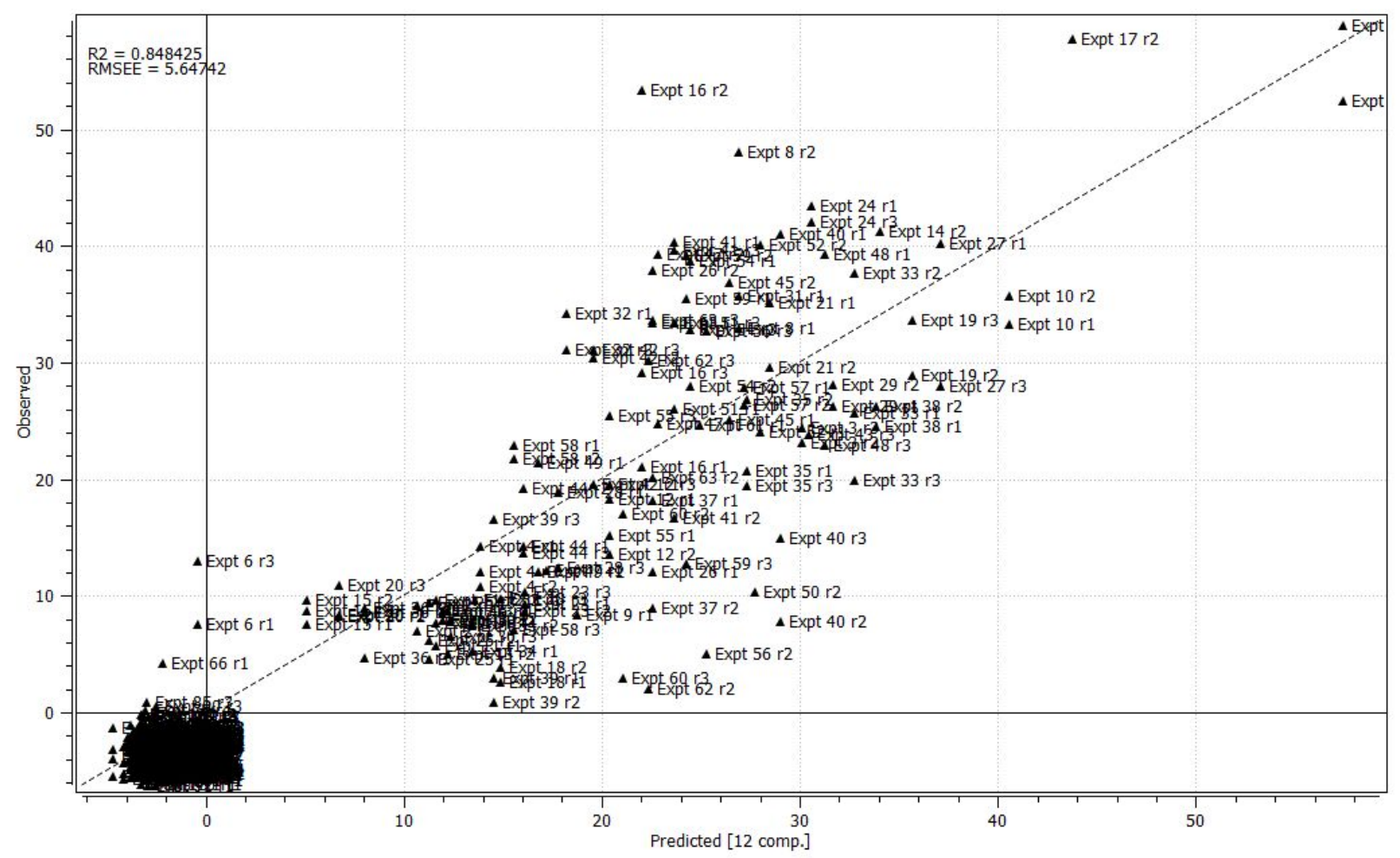

Figure S21. Observed (y-axis) versus predicted (x-axis) values for the burst release parameter $y_{o}$ based on the 12-component PLS model assembled for ovalbumin release from high-throughput fabricated hydrogels 


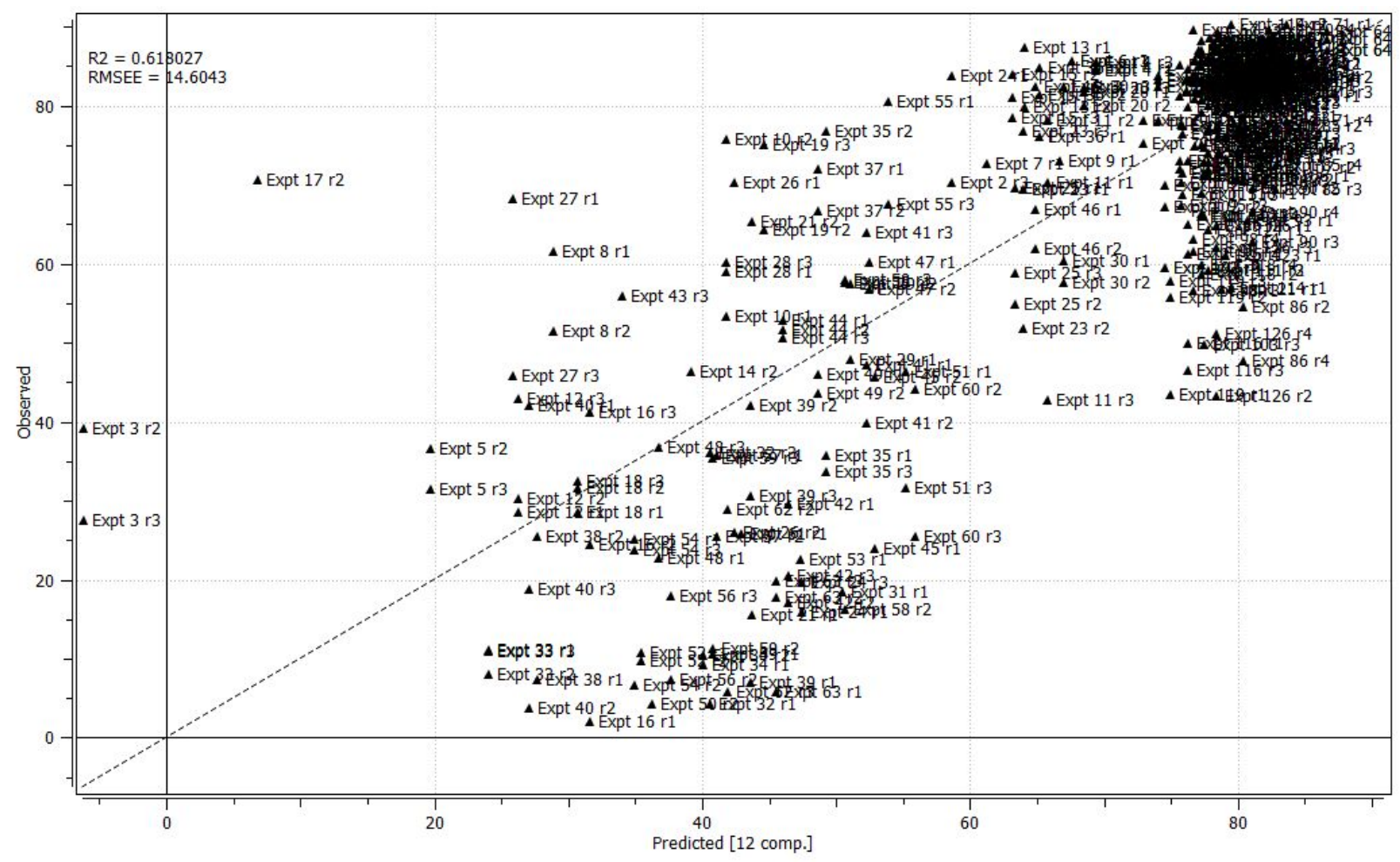

Figure S22. Observed (y-axis) versus predicted (x-axis) values for hydrogel transparency based on the 12-component PLS model 


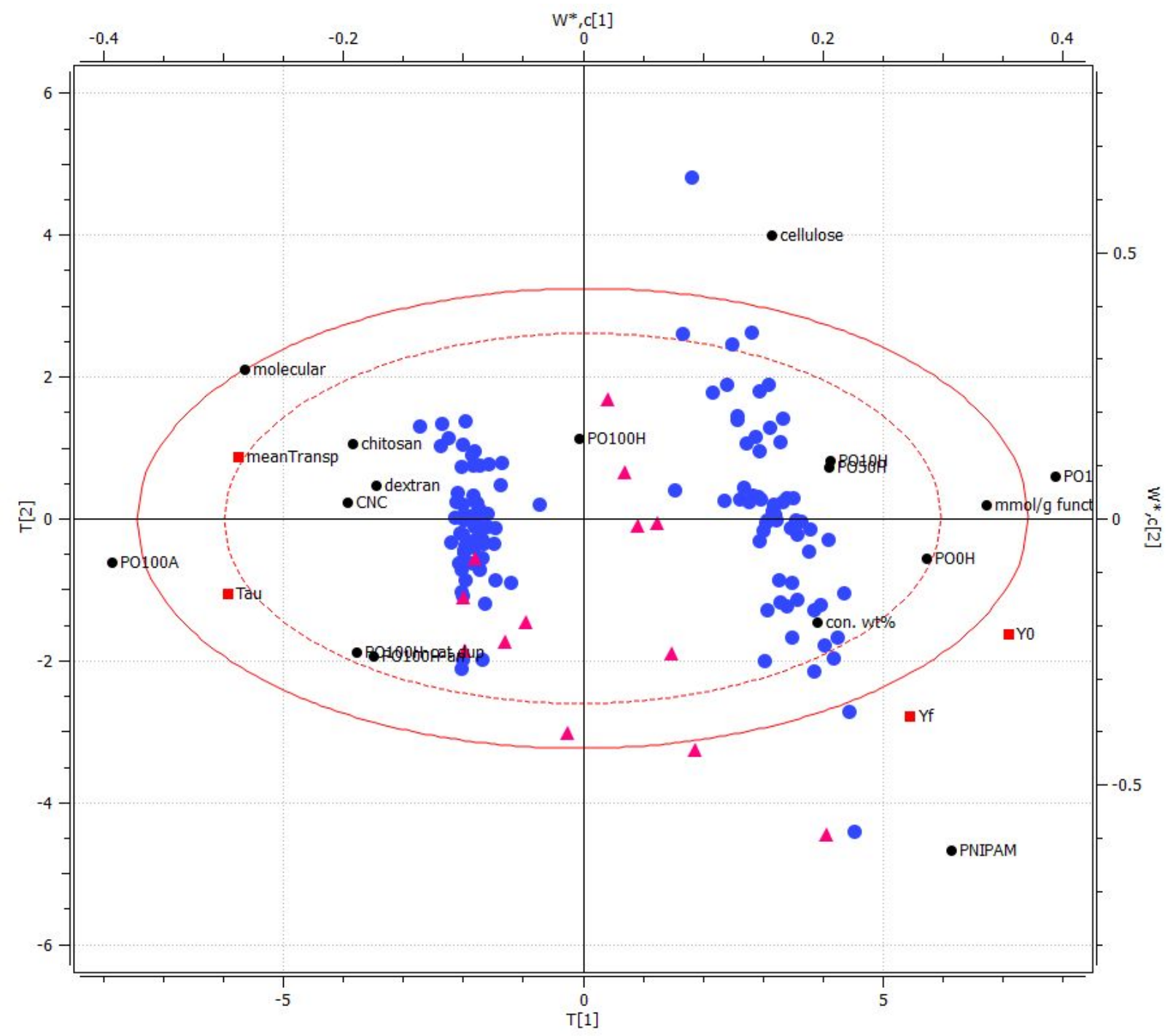

Figure S23. Loading bi-plot in the latent variable space correlating the input variables with the output variables. The blue dots represent each of the 126 hydrogels tested via high-throughput (i.e. the training data for the model), the red squares represent the key model fit parameters (e.g. transparency and the three drug release kinetics parameters), and the black dots represent the precursor polymers used to prepare the hydrogels. The pink triangles represent the location within the latent variable space in which the optimization samples were identified and run. Input variables (i.e. the precursor polymers and recipe parameters) that are close in space to output variables (i.e. transparency and the drug release parameters) are positively correlated while variables in opposite quadrants are negatively correlated. 